\title{
Anisotropic Singular Neumann Equations with Unbalanced Growth
}

\author{
Nikolaos S. Papageorgiou1 · Vicenţiu D. Rădulescu',3 (D) . Dušan D. Repovš ${ }^{4,5}$
}

Received: 27 October 2020 / Accepted: 24 January 2021 / Published online: 1 March 2021

(C) The Author(s) 2021

\begin{abstract}
We consider a nonlinear parametric Neumann problem driven by the anisotropic $(p, q)$ Laplacian and a reaction which exhibits the combined effects of a singular term and of a parametric superlinear perturbation. We are looking for positive solutions. Using a combination of topological and variational tools together with suitable truncation and comparison techniques, we prove a bifurcation-type result describing the set of positive solutions as the positive parameter $\lambda$ varies. We also show the existence of minimal positive solutions $u_{\lambda}^{*}$ and determine the monotonicity and continuity properties of the map $\lambda \mapsto u_{\lambda}^{*}$.
\end{abstract}

Keywords Modular function - Truncation - Comparison principle · Minimal solution . Anisotropic regularity

Mathematics Subject Classification (2010) $35 \mathrm{~J} 75 \cdot 35 \mathrm{~J} 60 \cdot 35 \mathrm{~J} 20$

This research was supported by the Slovenian Research Agency grants P1-0292, J1-8131, N1-0064, N1-0083, and N1-0114. The work of Vicenţiu D. Rădulescu was supported by a grant of the Romanian Ministry of Education and Research, CNCS-UEFISCDI, project number PN-III-P4-ID-PCE-2020-0068, within PNCDI III.

Vicenţiu D. Rădulescu

radulescu@inf.ucv.ro

Nikolaos S. Papageorgiou

npapg@math.ntua.gr

Dušan D. Repovš

dusan.repovs@guest.arnes.si

1 Department of Mathematics, National Technical University, Zografou Campus, 15780 Athens, Greece

2 Faculty of Applied Mathematics, AGH University of Science and Technology, 30-059 Krakow, Poland

3 Department of Mathematics, University of Craiova, 200585 Craiova, Romania

4 Faculty of Education and Faculty of Mathematics and Physics, University of Ljubljana, 1000 Ljubljana, Slovenia

5 Institute of Mathematics, Physics and Mechanics, 1000 Ljubljana, Slovenia 


\section{Introduction}

This paper was motivated by several recent contributions to the qualitative analysis of nonlinear problems with unbalanced growth. We mainly refer to the pioneering contributions of Marcellini [22-24] who studied lower semicontinuity and regularity properties of minimizers of certain quasiconvex integrals. Problems of this type arise in nonlinear elasticity and are connected with the deformation of an elastic body, cf. Ball [4, 5].

We are concerned with the qualitative analysis of a class of anisotropic singular problems with Neumann boundary condition and driven by a differential operator with unbalanced growth. The features of this paper are the following:

(i) the problem studied in the present work is associated to a double phase energy with variable exponents (variational integral with anisotropic unbalanced growth);

(ii) the reaction is both singular and anisotropic;

(iii) we assume a Neumann boundary condition.

To the best of our knowledge, this is the first paper dealing with the combined effects generated by the above features.

\subsection{Unbalanced Problems and Their Historical Traces}

Let $\Omega$ be a bounded domain in $\mathbb{R}^{N}(N \geq 2)$ with a smooth boundary. If $u: \Omega \rightarrow \mathbb{R}^{N}$ is the displacement and $\mathrm{Du}$ is the $N \times N$ matrix of the deformation gradient, then the total energy can be represented by an integral of the type

$$
I(u)=\int_{\Omega} f(z, D u(z)) d z,
$$

where the energy function $f=f(z, \xi): \Omega \times \mathbb{R}^{N \times N} \rightarrow \mathbb{R}$ is quasiconvex with respect to $\xi$, see Morrey [26]. One of the simplest examples considered by Ball is given by functions $f$ of the type

$$
f(\xi)=g(\xi)+h(\operatorname{det} \xi),
$$

where $\operatorname{det} \xi$ is the determinant of the $N \times N$ matrix $\xi$, and $g, h$ are nonnegative convex functions, which satisfy the growth conditions

$$
g(\xi) \geq c_{1}|\xi|^{p} ; \quad \lim _{t \rightarrow+\infty} h(t)=+\infty,
$$

where $c_{1}$ is a positive constant and $1<p<N$. The condition $p \leq N$ is necessary to study the existence of equilibrium solutions with cavities, that is, minima of the integral (1) that are discontinuous at one point where a cavity forms; in fact, every $u$ with finite energy belongs to the Sobolev space $W^{1, p}\left(\Omega, \mathbb{R}^{N}\right)$, and thus it is a continuous function if $p>N$. In accordance with these problems arising in nonlinear elasticity, Marcellini [22, 23 ] considered continuous functions $f=f(x, u)$ with unbalanced growth that satisfy

$$
c_{1}|u|^{p} \leq|f(z, u)| \leq c_{2}\left(1+|u|^{q}\right) \text { for all }(x, u) \in \Omega \times \mathbb{R},
$$

where $c_{1}, c_{2}$ are positive constants and $1 \leq p \leq q$. Regularity and existence of solutions of elliptic equations with $p, q$-growth conditions were studied in [23].

The study of non-autonomous functionals characterized by the fact that the energy density changes its ellipticity and growth properties according to the point has been continued in a series of remarkable papers by Mingione et al. [6, 7, 12]. These contributions are in relationship with the work of Zhikov [43, 44], which describe the behavior of phenomena arising in nonlinear elasticity. In fact, Zhikov intended to provide models for strongly 
anisotropic materials in the context of homogenisation. In particular, he considered the following model functional

$$
\mathcal{P}_{p, q}(u):=\int_{\Omega}\left(|D u|^{p}+a(z)|D u|^{q}\right) d z, \quad 0 \leq a(x) \leq L, 1<p<q,
$$

where the modulating coefficient $a(x)$ dictates the geometry of the composite made of two differential materials, with hardening exponents $p$ and $q$, respectively.

In the present paper we are concerned with a problem whose energy is of the type defined in Eq. 2 but such that the exponents $p$ and $q$ are variable (they depend on the point).

\subsection{Statement of the Problem}

Let $\Omega \subseteq \mathbb{R}^{N}$ be a bounded domain with a $C^{2}$-boundary $\partial \Omega$. In this paper we study the following parametric singular anisotropic $(p, q)$-equation:

$$
\left\{\begin{array}{l}
-\Delta_{p(z)} u(z)-\Delta_{q(z)} u(z)+\xi(z) u(z)^{p(z)-1}=u(z)^{-\eta(z)}+\lambda f(z, u(z)) \text { in } \Omega, \\
\frac{\partial u}{\partial n}=0 \text { on } \partial \Omega, u(z)>0 \text { for all } z \in \Omega, \lambda>0 .
\end{array}\right.
$$

In this problem, we make the following hypotheses for the exponents $p(\cdot), q(\cdot), \eta(\cdot)$ :

$$
p, q, \eta \in C^{1}(\bar{\Omega}), q_{-} \leq q_{+}<p_{-} \leq p_{+}, 0<\eta(z)<1 \text { for all } z \in \bar{\Omega},
$$

where for every $r \in C(\bar{\Omega})$ we define

$$
r_{-}:=\min _{\bar{\Omega}} r, r_{+}:=\max _{\bar{\Omega}} r
$$

Also for $r \in C(\bar{\Omega})$ with $1<r(z)<\infty$ for all $z \in \bar{\Omega}$, we denote by $\Delta_{r(z)}$ the $r(z)$ Laplace differential operator defined by

$$
\Delta_{r(z)} u=\operatorname{div}\left(|D u|^{r(z)-2} D u\right) \text { for all } u \in W^{1, r(z)}(\Omega) .
$$

The potential function $\xi \in L^{\infty}(\Omega)$ satisfies $\xi(z)>0$ for a.a. $z \in \Omega$. In the reaction we have two terms. One is the singular term $x \mapsto x^{-\eta(z)}$ with $0<\eta(z)<1$ for all $z \in \bar{\Omega}$ and the other is a parametric perturbation $\lambda f(z, x)$ with $\lambda>0$ being the parameter. The function $f(z, x)$ is a Carathéodory function, that is, for all $x \in \mathbb{R}$ the mapping $z \mapsto f(z, x)$ is measurable and for a.a. $z \in \Omega$ the function $x \mapsto f(z, x)$ is continuous. We assume that for a.a. $z \in \Omega$, the function $f(z, \cdot)$ exhibits a $\left(p_{+}-1\right)$-superlinear growth near $+\infty$ with $p_{+}=\max _{\bar{\Omega}} p$, but without satisfying the so-called Ambrosetti-Rabinowitz condition (the $A R$-condition for short), which is common in the literature when dealing with superlinear problems. Instead, we use a less restrictive condition which incorporates in our framework superlinear nonlinearities with slower growth near $+\infty$. The precise hypotheses on $f(z, x)$ can be found in Section 2 (see hypotheses $H_{1}$ ).

We are looking for positive solutions and our aim is to determine how the set of positive solutions changes as the parameter $\lambda>0$ varies. In this direction we prove a bifurcationtype result describing the changes in the set of positive solutions of $\left(P_{\lambda}\right)$ as the positive parameter $\lambda$ increases. We also show that if $\lambda>0$ is admissible (that is, problem $\left(P_{\lambda}\right)$ admits positive solutions), then there is a minimal positive solution $u_{\lambda}^{*}$ (that is, a smallest solution) and we examine the monotonicity and continuity of the map $\lambda \mapsto u_{\lambda}^{*}$.

Analogous studies for $p$-Laplacian equations with constant exponent, were conducted by Giacomoni et al. [18] and Papageorgiou and Winkert [28]. More general equations driven by nonhomogeneous differential operators, were considered recently by Papageorgiou et 
al. [33-36], Papageorgiou and Scapellato [27], Papageorgiou et al. [31], Papageorgiou and Zhang [30], and Ragusa and Tachikawa [38]. We should also mention the very recent works of De Filippis and Mingione [13] and Marcellini [25], on the regularity of solutions of double phase problems. This is a very interesting area with several issues remaining open and requiring further investigation. Finally, we mention the work of Bahrouni et al. [3] on a class of double phase problems with convection. Singular anisotropic equations driven by the $p(z)$-Laplacian, were studied by Byun and Ko [9], Zhang and Rădulescu [42], and Saudi and Ghanmi [40]. To the best of our knowledge, there are no works on singular anisotropic $(p, q)$-equations

Boundary value problems driven by a combination of differential operators (such as $(p, q)$-equations) arise in many mathematical models of physical processes. We mention the historically first such work of Cahn and Hilliard [10], which deals with the process of separation of binary alloys and the more recent works of Benci et al. [8] on quantum physics, of Cherfils and Ilyasov [11] on reaction diffusion systems and of Bahrouni et al. [1,2] on transonic flow problems. Boundary value problems involving differential operators with variable exponents, are studied in the book of Rădulescu and Repovš [39], while a comprehensive discussion of semilinear singular problems and a rich relevant bibliography can be found in the book of Ghergu and Rădulescu [17].

\section{Mathematical Background and Hypotheses}

Although as we already mentioned in the previous section, we require that our exponents $p(\cdot), q(\cdot), \eta(\cdot)$ are smooth (in order to exploit the existing anisotropic regularity theory), the introduction of the variable exponent spaces does not require such regularity restrictions.

We introduce the following spaces

$$
\begin{aligned}
& M(\Omega)=\{u: \Omega \rightarrow \mathbb{R} \text { measurable }\} \\
& L_{1}^{\infty}(\Omega)=\left\{p \in L^{\infty}(\Omega): 1 \leq \underset{\Omega}{\operatorname{essinf}} p\right\} .
\end{aligned}
$$

As usual, we identify in $M(\Omega)$ two functions which differ only on a set of measure zero. If $p \in L_{1}^{\infty}(\Omega)$, then we set

$$
p_{-}=\underset{\Omega}{\operatorname{essinf}} p \text { and } p_{+}=\underset{\Omega}{\operatorname{esssup}} p .
$$

Given $p \in L_{1}^{\infty}(\Omega)$, the variable exponent Lebesgue space $L^{p(z)}(\Omega)$ is defined by

$$
L^{p(z)}(\Omega)=\left\{u \in M(\Omega): \int_{\Omega}|u|^{p(z)} d z<\infty\right\} .
$$

We equip this space with the so-called "Luxemburg norm" defined by

$$
\|u\|_{p(z)}=\inf \left\{\lambda>0: \int_{\Omega}\left(\frac{|u|}{\lambda}\right)^{p(z)} d z \leq 1\right\} .
$$

Variable exponent Lebesgue spaces are similar to the classical Lebesgue spaces. More precisely, they are separable Banach spaces, they are reflexive if and only if $1<p_{-} \leq$ $p_{+}<\infty$ (in fact, they are uniformly convex). Moreover, simple functions and continuous functions of compact support are dense in $L^{p(z)}(\Omega)$.

Suppose that $p, q \in L_{1}^{\infty}(\Omega)$. Then we have the following property:

$$
\text { “ } L^{p(z)}(\Omega) \hookrightarrow L^{q(z)}(\Omega) \text { continuously }
$$




\section{if and only if \\ $q(z) \leq p(z)$ for a.a. $z \in \Omega$ ”.}

Let $p, p^{\prime} \in L_{1}^{\infty}(\Omega)$ such that $\frac{1}{p(z)}+\frac{1}{p^{\prime}(z)}=1$ for a.a. $z \in \Omega$. Then $L^{p(z)}(\Omega)^{*}=$ $L^{p^{\prime}(z)}(\Omega)$ and the following Hölder-type inequality is true

$$
\int_{\Omega}|u v| d z \leq\left(\frac{1}{p_{-}}+\frac{1}{p_{-}^{\prime}}\right)\|u\|_{p(z)}\|v\|_{p^{\prime}(z)}
$$

for all $u \in L^{p(z)}(\Omega)$ and all $v \in L^{p^{\prime}(z)}(\Omega)$.

Using the variable exponent Lebesgue spaces, we can define in the usual way variable exponent Sobolev spaces. So, if $p \in L_{1}^{\infty}(\Omega)$, then we set

$$
W^{1, p(z)}(\Omega)=\left\{u \in L^{p(z)}(\Omega):|D u| \in L^{p(z)}(\Omega)\right\} .
$$

This space is furnished with the following norm

$$
\|u\|=\|u\|_{p(z)}+\||D u|\|_{p(z)} .
$$

Evidently, an equivalent norm is given by

$$
|u|=\inf \left\{\lambda>0: \int_{\Omega}\left[\left(\frac{|D u|}{\lambda}\right)^{p(z)}+\left(\frac{|u|}{\lambda}\right)^{p(z)}\right] d z \leq 1\right\} .
$$

The anisotropic Sobolev space $W^{1, p(z)}(\Omega)$ is a separable Banach space and if $1<p_{-} \leq$ $p_{+}<\infty$, then $W^{1, p(z)}(\Omega)$ is reflexive (in fact, uniformly convex). Note that $W^{1, p(z)}(\Omega) \hookrightarrow$ $W^{1, p_{-}}(\Omega)$ continuously. Also, $W_{0}^{1, p(z)}(\Omega)$ is the closure of the set of $W^{1, p(z)}(\Omega)$-functions with compact support, that is, of the set

$$
\left\{u \in W^{1, p(z)}(\Omega): u=u \chi_{K} \text { with } K \subseteq \Omega \text { compact }\right\} .
$$

If $p \in C^{1}(\bar{\Omega})$, then $W_{0}^{1, p(z)}(\Omega)={\overline{C_{c}^{\infty}(\Omega)}}^{\|\cdot\|}$.

We set

$$
p^{*}(z)= \begin{cases}\frac{N p(z)}{N-p(z)}, & \text { if } p(z)<N \\ +\infty, & \text { if } N \leq p(z) .\end{cases}
$$

Suppose that $p, q \in L_{1}^{\infty}(\Omega) \cap C(\bar{\Omega})$ with $p_{+}<N$ and $q(z) \leq p^{*}(z)$ (resp., $q(z)<$ $\left.p^{*}(z)\right)$ for all $z \in \bar{\Omega}$, then we have that $W^{1, p(z)}(\Omega) \hookrightarrow L^{q(z)}(\Omega)$ continuously (resp., compactly). A comprehensive presentation of variable exponent Lebesgue and Sobolov spaces can be found in the book of Diening et al. [14].

Let $r \in L_{1}^{\infty}(\Omega)$ and consider the Lebesgue space $L^{r(z)}(\Omega)$. The modular function for this space is given by

$$
\rho_{r(z)}(u)=\int_{\Omega}|u|^{r(z)} d z .
$$

This function is basic in the study of $L^{r(z)}(\Omega)$ and is closely related to the norm $\|\cdot\|_{p(z)}$ introduced above. More specifically, we have the following result.

Proposition 2.1 (a) For $u \in L^{r(z)}(\Omega), u \neq 0$, we have

$$
\|u\|_{r(z)} \leq \lambda \Leftrightarrow \rho_{r(z)}\left(\frac{u}{\lambda}\right) \leq 1 ;
$$

(b) $\|u\|_{r(z)}<1($ resp. $=1,>1) \Leftrightarrow \rho_{r(z)}(u)<1($ resp. $=1,>1)$; 
(c) $\quad\|u\|_{r(z)}<1 \Rightarrow\|u\|_{r(z)}^{r_{+}} \leq \rho_{r(z)}(u) \leq\|u\|_{r(z)}^{r_{-}}$,

(d) $\|u\|_{r(z)}>1 \Rightarrow\|u\|_{r(z)}^{r_{-}} \leq \rho_{r(z)}(u) \leq\|u\|_{r(z)}^{r_{+}}$

(e) $\left\|u_{n}\right\|_{r(z)} \rightarrow+\infty \Leftrightarrow \rho_{r(z)}\left(u_{n}\right) \rightarrow+\infty$.

We consider the map $A_{r(z)}: W^{1, r(z)}(\Omega) \rightarrow W^{1, r(z)}(\Omega)^{*}$ defined by

$$
\left\langle A_{r(z)}(u), h\right\rangle=\int_{\Omega}|D u|^{r(z)-2}(D u, D h)_{\mathbb{R}^{N}} d z \text { for all } u, h \in W^{1, r(z)}(\Omega) .
$$

This map has the following properties (see Gasinski and Papageorgiou [16, Proposition 2.5]).

Proposition 2.2 The map $A_{r(z)}: W^{1, p(z)}(\Omega) \rightarrow W^{1, p(z)}(\Omega)^{*}$ is bounded (that is, maps bounded sets to bounded sets), continuous, monotone, hence also maximal monotone and of type $(S)_{+}$, that is,

$$
\text { " } u_{n} \stackrel{w}{\rightarrow} u \text { in } W^{1, r(z)}(\Omega), \limsup _{n \rightarrow \infty}\left\langle A_{r(z)}\left(u_{n}\right), u_{n}-u\right\rangle \leq 0 \Rightarrow u_{n} \rightarrow u \text { in } W^{1, r(z)}(\Omega) . "
$$

In addition to the variable exponent spaces, we will use the Banach space $C^{1}(\bar{\Omega})$. This is an ordered Banach space with positive cone $C_{+}=\left\{u \in C^{1}(\bar{\Omega}): u(z) \geq 0\right.$ for all $\left.z \in \bar{\Omega}\right\}$. This cone has nonempty interior given by

$$
\text { int } C_{+}=\left\{u \in C_{+}: u(z)>0 \text { for all } z \in \bar{\Omega}\right\} .
$$

We will also use another open cone in $C^{1}(\bar{\Omega})$ given by

$$
D_{+}=\left\{u \in C^{1}(\bar{\Omega}): u(z)>0 \text { for all } z \in \Omega,\left.\frac{\partial u}{\partial n}\right|_{\partial \Omega \cap u^{-1}(0)}<0\right\},
$$

with $n(\cdot)$ being the outward unit normal on $\partial \Omega$.

Combining the proofs of Proposition 2.5 of [37] and of Proposition 6 in [36] we have the following strong comparison principle.

Proposition 2.3 If $p, q, \eta \in C^{1}(\bar{\Omega}), 1<q_{-} \leq q_{+}<p_{-} \leq p_{+}, 0<\eta(z)<1$ for all $z \in \bar{\Omega}, \hat{\xi}, h, g \in L^{\infty}(\Omega), \hat{\xi}(z) \geq 0$ for a.a. $z \in \bar{\Omega}, 0<\mu \leq g(z)-h(z)$ for a.a. $z \in \Omega$ and $u, v \in C^{1}(\bar{\Omega})$ satisfy $0 \leq u \leq v$ and

$$
\begin{aligned}
& -\Delta_{p(z)} u-\Delta_{q(z)} u+\hat{\xi}(z) u^{p(z)-1}-u^{-\eta(z)}=h(z) \text { in } \Omega, \\
& -\Delta_{p(z)} v-\Delta_{q(z)} v+\hat{\xi}(z) v^{p(z)-1}-v^{-\eta(z)}=g(z) \text { in } \Omega,
\end{aligned}
$$

then $v-u \in D_{+}$.

If $u, v \in W^{1, p(z)}(\Omega)\left(p \in L_{1}^{\infty}(\Omega)\right)$ with $u \leq v$, then we define

$$
[u, v]=\left\{h \in W^{1, p(z)}(\Omega): u(z) \leq h(z) \leq v(z) \text { for a.a. } z \in \Omega\right\}
$$

and

$$
[u)=\left\{h \in W^{1, p(z)}(\Omega): u(z) \leq h(z) \text { for a.a. } z \in \Omega\right\} .
$$

If $X$ is a Banach space and $\varphi \in C^{1}(X, \mathbb{R})$, then we denote by $K_{\varphi}$ the critical set of $\varphi$, that is, the set

$$
K_{\varphi}=\left\{u \in X: \varphi^{\prime}(u)=0\right\} .
$$


Also, we say that $\varphi \in C^{1}(X, \mathbb{R})$ satisfies the " $C$-condition", if the following property holds:

"Every sequence $\left\{u_{n}\right\}_{n \geq 1} \subseteq X$ such that $\left\{\varphi\left(u_{n}\right)\right\}_{n \geq 1} \subseteq \mathbb{R}$ is bounded and and $\left(1+\left\|u_{n}\right\|_{X}\right) \varphi^{\prime}\left(u_{n}\right) \rightarrow 0$ in $X^{*}$ as $n \rightarrow \infty$, admits a strongly convergent subsequence".

This is a compactness-type condition on the functional $\varphi(\cdot)$. It compensates for the fact that $X$ is not locally compact, being in general infinite dimensional. The $C$-condition plays a crucial role in the minimax theory of the critical values of the functional $\varphi(\cdot)$.

Now we are ready to introduce the hypotheses on the data of $\left(P_{\lambda}\right)$.

$H_{0}: p, q, \eta \in C^{1}(\bar{\Omega}), 1<q_{-} \leq q_{+}<p_{-} \leq p_{+}, 0<\eta(z)<1$ for all $z \in \bar{\Omega}$, $\xi \in L^{\infty}(\Omega), \xi(z)>0$ for a.a. $z \in \Omega$.

$H_{1}: f: \Omega \times \mathbb{R} \rightarrow \mathbb{R}$ is a Carathéodory function (that is, $f(z, x)$ is measurable in $z \in \Omega$ and continuous in $x \in \mathbb{R})$ such that $f(z, 0)=0$ for a.a. $z \in \Omega$ and

(i) $0 \leq f(z, x) \leq a(z)\left[1+x^{r(z)-1}\right]$ for a.a. $z \in \Omega$, all $x \geq 0$ with $a \in L^{\infty}(\Omega)$ and $r \in C(\bar{\Omega})$ with $p_{+}<r_{-} \leq r_{+}<p^{*}(z)$ for all $z \in \bar{\Omega}$;

(ii) if $F(z, x)=\int_{0}^{x} f(z, s) d s$, then $\lim _{x \rightarrow+\infty} \frac{F(z, x)}{x^{p_{+}}}=+\infty$ uniformly for a.a. $z \in \Omega$;

(iii) if $\xi_{\lambda}(z, x)=\left(1-\frac{p_{+}}{1-\eta(z)}\right) x^{1-\eta(z)}+\lambda\left[f(z, x) x-p_{+} F(z, x)\right]$, then there exists $\hat{\vartheta}_{\lambda} \in L^{1}(\Omega)$ such that

$$
\xi_{\lambda}(z, x) \leq \xi_{\lambda}(z, y)+\hat{\vartheta}_{\lambda}(z) \text { for a.a. } z \in \Omega, \text { all } 0 \leq x \leq y ;
$$

(iv) for every $s>0$, we can find $\mu_{s}>0$ such that

$$
\begin{gathered}
0<\hat{\mu}_{s} \leq f(z, x) \text { for a.a. } z \in \Omega \text {, all } s \leq x \\
\text { and } 0<\hat{c} \leq \liminf _{x \rightarrow 0^{+}} \frac{f(z, x)}{x^{q_{+}-1}} \text { uniformly for a.a. } z \in \Omega ;
\end{gathered}
$$

(v) for every $\rho>0$, there exists $\hat{\xi}_{\rho}>0$ such that for a.a. $z \in \Omega$, the function

$$
x \mapsto f(z, x)+\hat{\xi}_{\rho} x^{p(z)-1}
$$

is nondecreasing on $[0, \rho]$.

Remarks Since we are looking for positive solutions and all the above hypotheses concern the positive semiaxis $\mathbb{R}_{+}=[0, \infty)$, without any loss of generality, we may assume that

$$
f(z, x)=0 \text { for a.a. } z \in \Omega \text {, all } x \leq 0 .
$$

On account of hypotheses $H_{1}(\mathrm{ii})$, (iii) we see that for a.a. $z \in \Omega, f(z, \cdot)$ is $\left(p_{+}-\right.$ 1)-superlinear. However the superlinearity property of $f(z, \cdot)$ is not expressed in terms of $A R$-condition. We recall that in the present anisotropic setting the $A R$-condition says that there exist $\vartheta>p_{+}$and $M>0$ such that

$$
\begin{aligned}
& 0<\vartheta F(z, x) \leq f(z, x) x \text { for a.a. } z \in \Omega \text {, all } x \geq M, \\
& 0<\underset{\Omega}{\operatorname{essinf}} F(\cdot, M) .
\end{aligned}
$$

In fact this is a unilateral version of the $A R$-condition due to Eq. 3. Integrating Eq. 4 and using Eq. 5, we obtain the weaker condition

$$
\begin{aligned}
& C_{0} x^{\vartheta} \leq F(z, x) \text { for a.a. } z \in \Omega, \text { all } x \geq M \text {, some } C_{0}>0, \\
\Rightarrow & C_{0} x^{\vartheta-1} \leq f(z, x) \text { for a.a. } z \in \Omega, \text { all } x \geq M .
\end{aligned}
$$


So, the $A R$-condition implies that $f(z, \cdot)$ eventually has $(\vartheta-1)$-polynomial growth. Here we replace the $A R$-condition by the quasimonotonicity hypothesis $H_{1}(\mathrm{iii})$. This hypothesis is a slightly more general version of a condition used by Li and Yang [20]. Note that there exists $M>0$ such that for a.a. $z \in \Omega$

$$
\begin{aligned}
x & \mapsto \frac{x^{-\eta(z)}+\lambda f(z, x)}{x^{p_{+}-1}} \text { is nondecreasing on } x \geq M \\
\text { or } x & \mapsto d_{\lambda}(z, x) \text { is nondecreasing on } x \geq M .
\end{aligned}
$$

Examples Consider the following two functions

$$
f_{1}(z, x)=\left(x^{+}\right)^{r(z)-1} \text { and } f_{2}(z, x)=\left(x^{+}\right)^{p(z)-1} \ln \left(1+x^{+}\right) .
$$

Both functions satisfy hypothesis $H_{1}$, but only $f_{1}$ satisfies the $A R$-condition.

By $L^{p(z)}(\Omega, \xi)$ we will denote the weighted $L^{p(z)}$-space with weight $\xi(\cdot)$. Therefore

$$
L^{p(z)}(\Omega, \xi)=\left\{u \in M(\Omega): \int_{\Omega} \xi(z)|u|^{p(z)} d z<\infty\right\} .
$$

This space is furnished with the norm

$$
\|u\|_{L^{p(z)}(\Omega, \xi)}=\inf \left\{\lambda>0: \int_{\Omega} \xi(z)\left|\frac{u}{\lambda}\right|^{p(z)} d z \leq 1\right\} .
$$

Note that since by hypothesis $\xi(z)>0$ for a.a. $z \in \Omega$ (see hypothesis $H_{0}$ ), the function

$$
\rho_{p(z), \xi}(u)=\int_{\Omega} \xi(z)|u|^{p(z)} d z,
$$

is a modular function (see Diening et al. [14, Definition 2.1.1, p. 20]).

On $W^{1, p(z)}(\Omega)$ we consider the norm $\|\cdot\|$ defined earlier and a new norm given by

$$
|u|=\|D u\|_{p(z)}+\|u\|_{L^{p(z)}(\Omega, \xi)} .
$$

Proposition 2.4 If hypothesis $H_{0}$ holds, then $\|\cdot\|$ and $|\cdot|$ are equivalent norms on $W^{1, p(z)}(\Omega)$.

Proof From the definitions of the two norms, we have

$$
|u| \leq C_{1}\|u\| \text { for some } C_{1}>0 \text {, all } u \in W^{1, p(z)}(\Omega) .
$$

Claim. There exists $C_{2}>0$ such that $\|u\|_{p(z)} \leq C_{2}|u|$ for all $u \in W^{1, p(z)}(\Omega)$.

We argue indirectly. So, suppose that the claim is not true. Then we can find $\left\{u_{n}\right\}_{n \geq 1} \subseteq$ $W^{1, p(z)}(\Omega)$ such that

$$
\left\|u_{n}\right\|_{p(z)}>n\left|u_{n}\right| \text { for all } n \in \mathbb{N} .
$$

Normalizing in $L^{p(z)}(\Omega)$, we see that we have

$$
\begin{aligned}
& \left|u_{n}\right|<\frac{1}{n} \text { for all } n \in \mathbb{N}, \\
\Rightarrow & \left|u_{n}\right| \rightarrow 0, \\
\Rightarrow & \left\|D u_{n}\right\|_{p(z)} \rightarrow 0 \text { and }\left\|u_{n}\right\|_{L^{p(z)}(\Omega, \xi)} \rightarrow 0 \text { as } n \rightarrow \infty .
\end{aligned}
$$

Evidently $\left\{u_{n}\right\}_{n \geq 1} \subseteq W^{1, p(z)}(\Omega)$ is bounded. So, by passing to a suitable subsequence if necessary, we may assume that

$$
\begin{aligned}
& u_{n} \stackrel{w}{\rightarrow} u \text { in } W^{1, p(z)}(\Omega) \text { and } u_{n} \rightarrow u \text { in } L^{p(z)}(\Omega), \\
\Rightarrow & \|u\|_{p(z)}=1 .
\end{aligned}
$$


From Eqs. 6 and 7, we have $u=\tilde{c} \in \mathbb{R} \backslash\{0\}$ and

$$
\int_{\Omega} \xi(z)\left|u_{n}\right|^{p(z)} d z \rightarrow \int_{\Omega} \xi(z)|\tilde{c}|^{p(z)} d z .
$$

On account of Eq. 6, we have

- If $0<|\tilde{c}| \leq 1$, then $0<|\tilde{c}|^{p_{+}} \int_{\Omega} \xi(z) d z \leq 0$, a contradiction.

- If $1<|\tilde{c}|$, then $0 \leq|\tilde{c}|^{p_{-}} \int_{\Omega} \xi(z) d z \leq 0$, a contradiction.

This proves the Claim.

Using the Claim and the definitions of the two norms, we conclude that $\|\cdot\|$ and $|\cdot|$ are equivalent.

In what follows, we denote by $\gamma_{p(z)}: W^{1, p(z)}(\Omega) \rightarrow \mathbb{R}$ the $C^{1}$-functional defined by

$$
\gamma_{p(z)}(u)=\int_{\Omega} \frac{1}{p(z)}|D u|^{p(z)} d z+\int_{\Omega} \frac{\xi(z)}{p(z)}|u|^{p(z)} d z \text { for every } u \in W^{1, p(z)}(\Omega) .
$$
by

For every $\lambda>0$ the energy functional $\varphi_{\lambda}: W^{1, p(z)}(\Omega) \rightarrow \mathbb{R}$ for problem $\left(P_{\lambda}\right)$, is given

$$
\varphi_{\lambda}(u)=\gamma_{p(z)}(u)+\int_{\Omega} \frac{1}{q(z)}|D u|^{q(z)} d z-\int_{\Omega} \frac{1}{1-\eta(z)}\left(u^{+}\right)^{1-\eta(z)} d z-\int_{\Omega} F\left(z, u^{+}\right) d z
$$

for all $u \in W^{1, p(z)}(\Omega)$.

On account of the third term, this functional is not $C^{1}$ and so the minimax theorems from the critical point theory are not directly applicable to this functional. For this reason we use truncation techniques in order to bypass the singularity and have $C^{1}$-functionals on which the critical point theory applies. For this reason in the next section, we deal with a purely singular problem.

Finally, we mention that, as usual, by a solution of $\left(P_{\lambda}\right)$, we understand a function $u \in$ $W^{1, p(z)}(\Omega)$ such that

$$
u \geq 0, u \not \equiv 0, u^{-\eta(\cdot)} h \in L^{1}(\Omega) \text { for all } h \in W^{1, p(z)}(\Omega)
$$

and

$$
\begin{aligned}
& \left\langle A_{p(z)}(u), h\right\rangle+\left\langle A_{q(z)}(u), h\right\rangle+\int_{\Omega} \xi(z) u^{p(z)-1} h d z \\
= & \int_{\Omega}\left[u^{-\eta(z)}+\lambda f(z, u)\right] h d z \text { for all } h \in W^{1, p(z)}(\Omega) .
\end{aligned}
$$

\section{A Purely Singular Problem}

In this section we deal with the following purely singular problem

$$
\left\{\begin{array}{l}
-\Delta_{p(z)} u(z)-\Delta_{q(z)} u(z)+\xi(z) u(z)^{p(z)-1}=u(z)^{-\eta(z)} \text { in } \Omega \\
\frac{\partial u}{\partial n}=0 \text { on } \partial \Omega, u>0 .
\end{array}\right.
$$


To solve Eq. 8, we first consider a perturbation of Eq. 8 which removes the singularity. So, we consider the following approximation of problem Eq. 8:

$$
\left\{\begin{array}{l}
-\Delta_{p(z)} u(z)-\Delta_{q(z)} u(z)+\xi(z) u(z)^{p(z)-1}=[u(z)+\varepsilon]^{-\eta(z)} \text { in } \Omega, \\
\frac{\partial u}{\partial n}=0 \text { on } \partial \Omega, u>0 .
\end{array}\right.
$$

We solve this problem using a topological approach (fixed point theory). So, given $g \in$ $L^{p(z)}(\Omega), g \geq 0$ and $\varepsilon \in(0,1]$, we consider the following problem:

$$
\left\{\begin{array}{l}
-\Delta_{p(z)} u(z)-\Delta_{q(z)} u(z)+\xi(z) u(z)^{p(z)-1}=[g(z)+\varepsilon]^{-\eta(z)} \text { in } \Omega \\
\frac{\partial u}{\partial n}=0 \text { on } \partial \Omega, u>0
\end{array}\right.
$$

For this problem we have the following result.

Proposition 3.1 If hypotheses $H_{0}$ hold, problem Eq. 9 admits a unique solution $\tilde{u}_{\varepsilon} \in$ int $C_{+}$.

Proof Let $K_{p(z)}: L^{p(z)}(\Omega) \rightarrow L^{p^{\prime}(z)}(\Omega)$ be the map defined by

$$
K_{p(z)}(u)=|u|^{p(z)-2} u \text { for all } u \in L^{p(z)}(\Omega) .
$$

Evidently this map is bounded, continuous, strictly monotone. Then we consider the operator $V: W^{1, p(z)}(\Omega) \rightarrow W^{1, p(z)}(\Omega)^{*}$ defined by

$$
V(u)=A_{p(z)}(u)+A_{q(z)}(u)+\xi(z) K_{p(z)}(u) \text { for all } u \in W^{1, p(z)}(\Omega) .
$$

This operator is bounded, continuous, strictly monotone (thus, maximal monotone too). Also, if $u \in W^{1, p(z)}(\Omega)$, we have

$$
\begin{aligned}
\langle V(u), u\rangle= & \rho_{p(z)}(D u)+\rho_{q(z)}(D u)+\int_{\Omega} \xi(z)|u|^{p(z)} d z \\
\geq & \rho_{p(z)}(D u)+\int_{\Omega} \xi(z)|u|^{p(z)} d z \\
\geq & \|D u\|_{p(z)}+\|u\|_{L^{p(z)}(\Omega, \xi)}-1 \\
& \left(\text { see Corollary } 2 . 1 . 1 5 \text { of } \left[14, \text { p. 25] and recall that } \rho_{p(z), \xi}(\cdot)\right.\right. \text { is modular) } \\
\geq & C_{2}\|u\| \text { for some } C_{2}>0(\text { see Proposition } 2.4), \\
\Rightarrow & V(\cdot) \text { is coercive. }
\end{aligned}
$$

We know that a maximal monotone coercive operator is surjective (see Papageorgiou et al. [32, Corollary 2.8.7, p. 135]). Since $[g(\cdot)+\varepsilon]^{-\eta(\cdot)} \in L^{\infty}(\Omega)$, we can find $\tilde{u}_{\varepsilon} \in$ $W^{1, p(z)}(\Omega)$ such that

$$
V\left(\tilde{u}_{\varepsilon}\right)=[g(\cdot)+\varepsilon]^{-\eta(\cdot)} .
$$

Moreover, the strict monotonicity of $V(\cdot)$ implies that this solution is unique. Proposition 3.1 of Gasinski and Papageorgiou [16] implies that $\tilde{u}_{\varepsilon} \in L^{\infty}(\Omega)$. Then by Theorem 1.3 of Fan [15] (see also Lieberman [21]), we have that $\tilde{u}_{\varepsilon} \in C_{+} \backslash\{0\}$. Finally the anisotropic maximum principle of Zhang [41] implies that $\tilde{u}_{\varepsilon} \in$ int $C_{+}$.

Using Proposition 3.1 we can define the solution map $L_{\varepsilon}: L^{p(z)}(\Omega) \rightarrow L^{p(z)}(\Omega)$ for problem Eq. 9 by

$$
L_{\varepsilon}(g)=\tilde{u}_{\varepsilon} .
$$

Clearly, a fixed point of this map will be a solution for problem $\left(8_{\varepsilon}\right)$. 
Proposition 3.2 If hypotheses $H_{0}$ hold, then problem $\left(8_{\varepsilon}\right)$ admits a unique solution $\bar{u}_{\varepsilon} \in$ int $C_{+}$.

Proof From Proposition 3.1, we have

$$
\left\langle A_{p(z)}\left(\tilde{u}_{\varepsilon}\right), h\right\rangle+\left\langle A_{q(z)}\left(\tilde{u}_{\varepsilon}\right), h\right\rangle+\int_{\Omega} \xi(z) \tilde{u}_{\varepsilon}^{p(z)-1} h d z=\int_{\Omega}[g(z)+\varepsilon]^{-\eta(z)} h d z
$$

for all $h \in W^{1, p(z)}(\Omega)$.

In Eq. 11 we choose $h=\tilde{u}_{\varepsilon}=L_{\varepsilon}(g) \in W^{1, p(z)}(\Omega)$ (see Eq. 10) and we obtain

$$
\begin{aligned}
& \rho_{p(z)}\left(D \tilde{u}_{\varepsilon}\right)+\rho_{q(z)}\left(D \tilde{u}_{\varepsilon}\right)+\int_{\Omega} \xi(z) \tilde{u}_{\varepsilon}^{p(z)} d z=\int_{\Omega} \frac{\tilde{u}_{\varepsilon}}{[g(z)+\varepsilon]^{\eta(z)}} d z, \\
\Rightarrow & \left\{\begin{array}{l}
\left\|L_{\varepsilon}(g)\right\|^{p_{+}} \leq C_{3}\left\|L_{\varepsilon}(g)\right\| \text { if }\left\|L_{\varepsilon}(g)\right\| \leq 1 \\
\left\|L_{\varepsilon}(g)\right\|^{p_{-}} \leq C_{3}\left\|L_{\varepsilon}(g)\right\| \text { if }\left\|L_{\varepsilon}(g)\right\|>1 \text { for some } C_{3}=C_{3}(\varepsilon)>0, \text { all } g \in L^{\infty}(\Omega)
\end{array}\right.
\end{aligned}
$$

(see Proposition 2.4).

Next, we show that $L_{\varepsilon}(\cdot)$ is continuous. To this end let $g_{n} \rightarrow g$ in $L^{p(z)}(\Omega)$. From Eq. 12 we have that

So, we may assume that

$$
\left\{L_{\varepsilon}\left(g_{n}\right)=\tilde{u}_{\varepsilon_{n}}=\tilde{u}_{n}\right\}_{n \geq 1} \subseteq W^{1, p(z)}(\Omega) \text { is bounded. }
$$

$$
\tilde{u}_{n} \stackrel{w}{\rightarrow} \tilde{u} \text { in } W^{1, p(z)}(\Omega) \text { and } \tilde{u}_{n} \rightarrow \tilde{u} \text { in } L^{p(z)}(\Omega) .
$$

We have

$$
\left\langle A_{p(z)}\left(\tilde{u}_{n}\right), h\right\rangle+\left\langle A_{q(z)}\left(\tilde{u}_{n}\right), h\right\rangle+\int_{\Omega} \xi(z)\left(\tilde{u}_{n}\right)^{p(z)-1} h d z=\int_{\Omega} \frac{h}{\left[g_{n}+\varepsilon\right]^{\eta(z)}} d z
$$

for all $h \in W^{1, p(z)}(\Omega)$, all $n \in \mathbb{N}$.

In Eq. 14 we choose $h=\tilde{u}_{n}-\tilde{u} \in W^{1, p(z)}(\Omega)$, pass to the limit as $n \rightarrow \infty$ and use Eq. 13. Then we have

$$
\begin{aligned}
& \lim _{n \rightarrow \infty}\left[\left\langle A_{p(z)}\left(\tilde{u}_{n}\right), \tilde{u}_{n}-\tilde{u}\right\rangle+\left\langle A_{q(z)}\left(\tilde{u}_{n}\right), \tilde{u}_{n}-\tilde{u}\right\rangle\right]=0, \\
\Rightarrow & \limsup _{n \rightarrow \infty}\left[\left\langle A_{p(z)}\left(\tilde{u}_{n}\right), \tilde{u}_{n}-\tilde{u}\right\rangle+\left\langle A_{q(z)}(\tilde{u}), \tilde{u}_{n}-\tilde{u}\right\rangle\right] \leq 0 \\
& \left(\text { since } A_{q(z)}(\cdot)\right. \text { is monotone), } \\
\Rightarrow & \limsup _{n \rightarrow \infty}\left\langle A_{p(z)}\left(\tilde{u}_{n}\right), \tilde{u}_{n}-\tilde{u}\right\rangle \leq 0 \text { (see Eq. 13), } \\
\Rightarrow & \tilde{u}_{n} \rightarrow \tilde{u} \text { in } W^{1, p(z)}(\Omega) \text { (see Proposition 2.2). }
\end{aligned}
$$

If in Eq. 14 we pass to the limit as $n \rightarrow \infty$ and use Eq. 15, we obtain that

$$
\begin{aligned}
& \left\langle A_{p(z)}(\tilde{u}), h\right\rangle+\left\langle A_{q(z)}(\tilde{u}), h\right\rangle+\int_{\Omega} \xi(z) \tilde{u}^{p(z)-1} h d z=\int_{\Omega} \frac{h}{[g+\varepsilon]^{\eta(z)}} d z \\
& \text { for all } h \in W^{1, p(z)}(\Omega) \\
\Rightarrow & \tilde{u}=L_{\varepsilon}(g) \\
\Rightarrow & L_{\varepsilon}(\cdot) \text { is continuous. }
\end{aligned}
$$

The continuity of $L_{\varepsilon}(\cdot)$ together with Eq. 12 and the compact embedding of $W^{1, p(z)}(\Omega)$ into $L^{p(z)}(\Omega)$, permit the use of Schauder-Tychonov fixed point theorem (see, for example, Papageorgiou and Winkert [29, Theorem 6.8.5, p. 581]) and we find $\bar{u}_{\varepsilon} \in \operatorname{int} C_{+}$such that

$$
\begin{aligned}
& L_{\varepsilon}\left(\bar{u}_{\varepsilon}\right)=\bar{u}_{\varepsilon}, \\
\Rightarrow & \bar{u}_{\varepsilon} \in \operatorname{int} C_{+} \text {is a positive solution of Eq. } 8 .
\end{aligned}
$$


Next we show the uniqueness of this solution. Suppose that $\hat{u}_{\varepsilon} \in W^{1, p(z)}(\Omega)$ is another positive solution of Eq. 8. Again we have $\hat{u}_{\varepsilon} \in$ int $C_{+}$. Also, we have

$$
\begin{aligned}
0 & \leq\left\langle A_{p(z)}\left(\bar{u}_{\varepsilon}\right)-A_{p(z)}\left(\hat{u}_{\varepsilon}\right),\left(\bar{u}_{\varepsilon}-\hat{u}_{\varepsilon}\right)^{+}\right\rangle+\left\langle A_{q(z)}\left(\bar{u}_{\varepsilon}\right)-A_{q(z)}\left(\hat{u}_{\varepsilon}\right),\left(\bar{u}_{\varepsilon}-\hat{u}_{\varepsilon}\right)^{+}\right\rangle \\
& +\int_{\Omega} \xi(z)\left(\bar{u}_{\varepsilon}^{p(z)-1}-\hat{u}_{\varepsilon}^{p(z)-1}\right)\left(\bar{u}_{\varepsilon}-\hat{u}_{\varepsilon}\right)^{+} d z= \\
& =\int_{\Omega}\left[\frac{1}{\left[\bar{u}_{\varepsilon}+\varepsilon\right]^{\eta(z)}}-\frac{1}{\left[\hat{u}_{\varepsilon}+\varepsilon\right]^{\eta(z)}}\right]\left(\bar{u}_{\varepsilon}-\hat{u}_{\varepsilon}\right)^{+} d z \leq 0 \\
\Rightarrow \bar{u}_{\varepsilon} & \leq \hat{u}_{\varepsilon} .
\end{aligned}
$$

Interchanging the roles of $\bar{u}_{\varepsilon}$ and $\hat{u}_{\varepsilon}$ in the above argument, we also have that $\hat{u}_{\varepsilon} \leq \bar{u}_{\varepsilon}$, therefore $\bar{u}_{\varepsilon}=\hat{u}_{\varepsilon}$. This proves the uniqueness of the positive solution $\bar{u}_{\varepsilon} \in \operatorname{int} C_{+}$of problem $\left(8_{\varepsilon}\right)$.

Evidently, to produce a positive solution of Eq. 8, we will let $\varepsilon \rightarrow 0^{+}$.

To this end, the following monotonicity property of the map $\varepsilon \mapsto \bar{u}_{\varepsilon}$ will be useful.

Proposition 3.3 If hypotheses $H_{0}$ hold, then the map $\varepsilon \mapsto \bar{u}_{\varepsilon}$ from $(0,1]$ into $C_{+}$is nonincreasing, that is,

$$
0<\varepsilon^{\prime}<\varepsilon \leq 1 \Rightarrow \bar{u}_{\varepsilon} \leq \bar{u}_{\varepsilon^{\prime}}
$$

Proof Let $0<\varepsilon^{\prime}<\varepsilon \leq 1$ and consider $\bar{u}_{\varepsilon^{\prime}}, \bar{u}_{\varepsilon} \in$ int $C_{+}$the corresponding unique positive solutions of problems $\left(8_{\varepsilon^{\prime}}\right)$ and Eq. $8_{\varepsilon}$ respectively, established in Proposition 3.2.

We have

$$
\begin{aligned}
& -\Delta_{p(z)} \bar{u}_{\varepsilon^{\prime}}-\Delta_{q(z)} \bar{u}_{\varepsilon^{\prime}}+\xi(z) \bar{u}_{\varepsilon^{\prime}}^{p(z)-1} \\
= & {\left[\bar{u}_{\varepsilon^{\prime}}+\varepsilon^{\prime}\right]^{-\eta(z)} } \\
\geq & {\left[\bar{u}_{\varepsilon^{\prime}}+\varepsilon\right]^{-\eta(z)} \text { in } \Omega\left(\text { since } 0<\varepsilon^{\prime}<\varepsilon\right) . }
\end{aligned}
$$

We introduce the Carathéodory function $e_{\varepsilon}(z, x)$ defined by

$$
e_{\varepsilon}(z, x)= \begin{cases}\frac{1}{\left[x^{+}+\varepsilon\right]^{\eta(z)}}, & \text { if } x \leq \bar{u}_{\mathcal{\varepsilon}^{\prime}}(z) \\ \frac{1}{\left[\bar{u}_{\mathcal{E}^{\prime}}(z)+\varepsilon\right]^{\eta(z)}}, & \text { if } \bar{u}_{\varepsilon^{\prime}}(z)<x .\end{cases}
$$

We set $E_{\varepsilon}(z, x)=\int_{0}^{x} e_{\varepsilon}(z, s) d s$ and consider the $C^{1}$-functional $\tau_{\varepsilon}: W^{1, p(z)}(\Omega) \rightarrow \mathbb{R}$ defined by

$$
\tau_{\varepsilon}(u)=\gamma_{p(z)}(u)+\int_{\Omega} \frac{1}{q(z)}|D u|^{q(z)} d z-\int_{\Omega} E_{\varepsilon}(z, u) d z \text { for all } u \in W^{1, p(z)}(\Omega) .
$$

We have

$$
\begin{aligned}
\tau_{\varepsilon}(u) & \geq \rho_{p(z)}(D u)+\rho_{p(z), \xi}(u)-C_{4} \text { for some } C_{4}>0 \text { (see Eq. 17) } \\
& \geq|u|-C_{4}-1 \\
\Rightarrow \tau_{\varepsilon}(\cdot) & \text { is coercive (see Proposition 2.4). }
\end{aligned}
$$

Also, exploiting the compact embedding of $W^{1, p(z)}(\Omega)$ into $L^{p(z)}(\Omega)$, we have that $\tau_{\varepsilon}(\cdot)$ is sequentially weakly lower semicontinuous. 
Invoking the Weierstrass-Tonelli theorem, we can find $\hat{u}_{\varepsilon} \in W^{1, p(z)}(\Omega)$ such that

$$
\begin{gathered}
\tau_{\varepsilon}\left(\hat{u}_{\varepsilon}\right)=\min \left\{\tau_{\varepsilon}(u): u \in W^{1, p(z)}(\Omega)\right\}, \\
\Rightarrow \tau_{\varepsilon}^{\prime}\left(\hat{u}_{\varepsilon}\right)=0, \\
\Rightarrow\left\langle A_{p(z)}\left(\hat{u}_{\varepsilon}\right), h\right\rangle+\left\langle A_{q(z)}\left(\hat{u}_{\varepsilon}\right), h\right\rangle+\int_{\Omega} \xi(z)\left|\hat{u}_{\varepsilon}\right|^{p(z)-2} \hat{u}_{\varepsilon} h d z=\int_{\Omega} e_{\varepsilon}\left(z, \hat{u}_{\varepsilon}\right) h d z
\end{gathered}
$$

for all $h \in W^{1, p(z)}(\Omega)$.

In Eq. 18 we choose $h=-\hat{u}_{\varepsilon}^{-} \in W^{1, p(z)}(\Omega)$ and obtain

$$
\begin{aligned}
& \rho_{p(z)}\left(D \hat{u}_{\varepsilon}^{-}\right)+\rho_{q(z)}\left(D \hat{u}_{\varepsilon}^{-}\right)+\rho_{p(z), \xi}\left(\hat{u}_{\varepsilon}^{-}\right)=0 \text { (see Eq. 17), } \\
\Rightarrow & \rho_{p(z)}\left(D u_{\varepsilon}^{-}\right)+\rho_{p(z), \xi}\left(\hat{u}_{\varepsilon}^{-}\right) \leq 0 \\
\Rightarrow & \hat{u}_{\varepsilon} \geq 0, \hat{u}_{\varepsilon} \neq 0 \text { (see Eq. 18). }
\end{aligned}
$$

Next, in Eq. 18 we choose $h=\left[\hat{u}_{\varepsilon}-\bar{u}_{\mathcal{\varepsilon}^{\prime}}\right]^{+} \in W^{1, p(z)}(\Omega)$. We obtain

$$
\begin{aligned}
& \left\langle A_{p(z)}\left(\hat{u}_{\varepsilon}\right),\left(\hat{u}_{\varepsilon}-\bar{u}_{\varepsilon^{\prime}}\right)^{+}\right\rangle+\left\langle A_{q(z)}\left(\hat{u}_{\varepsilon}\right),\left(\hat{u}_{\varepsilon}-\bar{u}_{\varepsilon^{\prime}}\right)^{+}\right\rangle+\int_{\Omega} \xi(z) \hat{u}_{\varepsilon}^{p(z)-1}\left(\hat{u}_{\varepsilon}-\bar{u}_{\varepsilon^{\prime}}\right)^{+} d z \\
= & \int_{\Omega} \frac{\left[\hat{u}_{\varepsilon}-\bar{u}_{\varepsilon^{\prime}}\right]^{+}}{\left[\bar{u}_{\varepsilon^{\prime}}+\varepsilon\right]^{\eta(z)}} d z \text { (see Eq. 17) } \\
\leq & \left\langle A_{p(z)}\left(\bar{u}_{\varepsilon^{\prime}}\right),\left(\hat{u}_{\varepsilon}-\bar{u}_{\varepsilon^{\prime}}\right)^{+}\right\rangle+\left\langle A_{q(z)}\left(\bar{u}_{\varepsilon^{\prime}}\right),\left(\hat{u}_{\varepsilon}-\bar{u}_{\varepsilon^{\prime}}\right)^{+}\right\rangle+\int_{\Omega} \xi(z) \bar{u}_{\varepsilon^{\prime}}^{p(z)-1}\left(\hat{u}_{\varepsilon}-\bar{u}_{\varepsilon^{\prime}}\right)^{+} d z, \\
\Rightarrow \hat{u}_{\varepsilon} \leq & \bar{u}_{\varepsilon^{\prime}} .
\end{aligned}
$$

So, we have proved that

$$
\begin{aligned}
& \hat{u}_{\varepsilon} \in\left[0, \bar{u}_{\varepsilon^{\prime}}\right], \hat{u}_{\varepsilon} \neq 0, \\
\Rightarrow & \hat{u}_{\varepsilon}=\bar{u}_{\varepsilon}(\text { see Eqs. 18, } 17 \text { and Proposition 3.2), } \\
\Rightarrow & \bar{u}_{\varepsilon} \leq \bar{u}_{\varepsilon^{\prime}} .
\end{aligned}
$$

The proof is now complete.

Now we will pass to the limit as $\varepsilon \rightarrow 0^{+}$and produce a solution for problem (8).

Proposition 3.4 If hypotheses $H_{0}$ hold, then problem Eq. 8 admits a unique solution $\bar{u} \in$ int $C_{+}$.

Proof Let $\left\{\varepsilon_{n}\right\}_{n \geq 1} \subseteq(0,1]$ be such that $\varepsilon_{n} \rightarrow 0^{+}$and let $\bar{u}_{n}=\bar{u}_{\varepsilon_{n}} \in$ int $C_{+}$be as in Proposition 3.2. We have

$$
\left\langle A_{p(z)}\left(\bar{u}_{n}\right), h\right\rangle+\left\langle A_{q(z)}\left(\bar{u}_{n}\right), h\right\rangle+\int_{\Omega} \xi(z) \bar{u}_{n}^{p(z)-1} h d z=\int_{\Omega} \frac{h}{\left[\bar{u}_{n}+\varepsilon_{n}\right]^{\eta(z)}} d z
$$

for all $h \in W^{1, p(z)}(\Omega)$, all $n \in \mathbb{N}$.

We choose $h=\bar{u}_{n} \in W^{1, p(z)}(\Omega)$. We obtain

$$
\rho_{p(z)}\left(D \bar{u}_{n}\right)+\rho_{q(z)}\left(D \bar{u}_{n}\right)+\int_{\Omega} \xi(z) \bar{u}_{n}^{p(z)} d z \leq \int_{\Omega} \frac{\bar{u}_{n}}{\bar{u}_{1}^{\eta(z)}}
$$

(since $\bar{u}_{1} \leq \bar{u}_{n}$ for all $n \in \mathbb{N}$, see Proposition 3.3)

$\Rightarrow\left\{\bar{u}_{n}\right\}_{n \geq 1} \subseteq W^{1, p(z)}(\Omega)$ is bounded

(see Proposition 2.4 and recall that $p_{-}>1$ ). 

that

Proposition 3.1 of Gasinski and Papageorgiou [16], implies that we can find $C_{5}>0$ such

$$
\left\|\bar{u}_{n}\right\|_{\infty} \leq C_{5} \text { for all } n \in \mathbb{N} .
$$

Then the anisotropic regularity theory of Fan [15, Theorem 1.3] implies that we can find $\alpha \in(0,1)$ and $C_{6}>0$ such that

$$
\bar{u}_{n} \in C^{1, \alpha}(\bar{\Omega}),\left\|\bar{u}_{n}\right\|_{C^{1, \alpha}(\bar{\Omega})} \leq C_{6} \text { for all } n \in \mathbb{N} .
$$

The compact embedding of $C^{1, \alpha}(\bar{\Omega})$ into $C^{1}(\bar{\Omega})$ and the monotonicity of the sequence $\left\{\bar{u}_{n}\right\}_{n \geq 1}$ (see Proposition 3.3), imply that

$$
\bar{u}_{n} \rightarrow \bar{u} \text { in } C^{1}(\bar{\Omega}) .
$$

Since $\bar{u}_{1} \leq \bar{u}_{n}$ for all $n \in \mathbb{N}$, we have $\bar{u} \neq 0$ and so $\bar{u} \in \operatorname{int} C_{+}$. Moreover, passing to the limit as $n \rightarrow \infty$ in Eq. 19 and using Eq. 20, we conclude that $\bar{u} \in \operatorname{int} C_{+}$is a positive solution of problem Eq. 8.

Finally, we show the uniqueness of this positive solution. So, suppose that $\tilde{u} \in$ $W^{1, p(z)}(\Omega)$ is another positive solution of Eq. 8. As in the proof of Proposition 3.2, using the fact that the map $x \mapsto x^{-\eta(z)}$ is strictly decreasing on $(0,+\infty)$, we obtain

$$
\begin{aligned}
& \tilde{u}=\bar{u}, \\
\Rightarrow \bar{u} & \in \operatorname{int} C_{+} \text {is the unique positive solution of Eq. } 8 .
\end{aligned}
$$

The proof is now complete.

In the next section we will use $\bar{u} \in$ int $C_{+}$and truncation techniques to bypass the singularity and show that problem $\left(P_{\lambda}\right)$ has positive solutions for certain values of the parameter $\lambda>0$.

\section{Positive Solutions}

We introduce the following two sets

$$
\begin{aligned}
& \mathcal{L}=\left\{\lambda>0 \text { : problem }\left(P_{\lambda}\right) \text { has a positive solution }\right\}, \\
& S_{\lambda}=\text { set of positive solutions of problem }\left(P_{\lambda}\right) .
\end{aligned}
$$

We start by showing the nonemptiness of $\mathcal{L}$ (=the set of admissible parameter values).

Proposition 4.1 If hypotheses $H_{0}, H_{1}$ hold, then $\mathcal{L} \neq \emptyset$.

Proof Let $\bar{u} \in \operatorname{int} C_{+}$be the unique positive solution of problem Eq. 8 produced in Proposition 3.4.

We consider the following auxiliary problem:

$$
\left\{\begin{array}{l}
-\Delta_{p(z)} u(z)-\Delta_{q(z)} u(z)+\xi(z) u(z)^{p(z)-1}=\bar{u}(z)^{-\eta(z)}+1 \text { in } \Omega \\
\frac{\partial u}{\partial n}=0 \text { on } \partial \Omega, u>0 .
\end{array}\right.
$$


From Proposition 3.1, we know that this problem has a unique positive solution $\hat{u} \in$ int $C_{+}$. We have

$$
\begin{aligned}
& \left\langle A_{p(z)}(\bar{u}),(\bar{u}-\hat{u})^{+}\right\rangle+\left\langle A_{q(z)}(\bar{u}),(\bar{u}-\hat{u})^{+}\right\rangle+\int_{\Omega} \xi(z) \bar{u}^{p(z)-1}(\bar{u}-\hat{u})^{+} d z \\
= & \int_{\Omega} \bar{u}^{-\eta(z)}(\bar{u}-\hat{u})^{+} d z \text { (see Eq. 8) } \\
\leq & \int_{\Omega}\left[\bar{u}^{-\eta(z)}+1\right](\bar{u}-\hat{u})^{+} d z \\
= & \left\langle A_{p(z)}(\hat{u}),(\bar{u}-\hat{u})^{+}\right\rangle+\left\langle A_{q(z)}(\hat{u}),(\bar{u}-\hat{u})^{+}\right\rangle+\int_{\Omega} \xi(z) \hat{u}^{p(z)-1}(\bar{u}-\hat{u})^{+} d z
\end{aligned}
$$

(since $\hat{u} \in \operatorname{int} C_{+}$solves Eq. 21),

$$
\Rightarrow \bar{u} \leq \hat{u}
$$

Since $\hat{u} \in$ int $C_{+}$, on account of hypothesis $H_{1}(i)$, we have

$$
0 \leq f(\cdot, \hat{u}(\cdot)) \in L^{\infty}(\Omega)
$$

So, we can find $\lambda_{0}>0$ such that

$$
0 \leq \lambda f(z, \hat{u}(z)) \leq 1 \text { for a.a. } z \in \Omega \text {, all } \lambda \in\left(0, \lambda_{0}\right]
$$

We introduce the Carathéodory function $\beta_{\lambda}(z, x)$ defined by

$$
\beta_{\lambda}(z, x)= \begin{cases}\bar{u}(z)^{-\eta(z)}+\lambda f(z, \bar{u}(z)), & \text { if } x<\bar{u}(z) \\ x^{-\eta(z)}+\lambda f(z, x), & \text { if } \bar{u}(z) \leq x \leq \hat{u}(z) \text { (see Eq. 22) } \\ \hat{u}(z)^{-\eta(z)}+\lambda f(z, \hat{u}(z)), & \text { if } \hat{u}(z)<x\end{cases}
$$

We set $B_{\lambda}(z, x)=\int_{0}^{x} \beta_{\lambda}(z, s) d s$ and consider the $C^{1}$-functional $\tilde{\Psi}_{\lambda}: W^{1, p(z)}(\Omega) \rightarrow \mathbb{R}$ defined by $\tilde{\Psi}_{\lambda}(u)=\gamma_{p(z)}(u)+\int_{\Omega} \frac{1}{q(z)}|D u|^{q(z)} d z-\int_{\Omega} B_{\lambda}(z, u) d z$ for all $u \in W^{1, p(z)}(\Omega)$, all $\lambda \in\left(0, \lambda_{0}\right]$.

From Eq. 22 it is clear that $\tilde{\Psi}_{\lambda}(\cdot)$ is coercive. Also, it is sequentially weakly lower semicontinuous. Hence, we can find $\tilde{u} \in W^{1, p(z)}(\Omega)$ such that

$$
\begin{gathered}
\tilde{\Psi}_{\lambda}(\tilde{u})=\min \left\{\tilde{\Psi}_{\lambda}(u): u \in W^{1, p(z)}(\Omega)\right\}, \\
\Rightarrow \tilde{\Psi}_{\lambda}^{\prime}(\tilde{u})=0, \\
\Rightarrow\left\langle A_{p(z)}(\tilde{u}), h\right\rangle+\left\langle A_{q(z)}(\tilde{u}), h\right\rangle+\int_{\Omega} \xi(z)|\tilde{u}|^{p(z)-2} \tilde{u} h d z=\int_{\Omega} \beta_{\lambda}(z, \tilde{u}) h d z
\end{gathered}
$$

for all $h \in W^{1, p(z)}(\Omega)$. 
In Eq. 25 first we choose $h=(\bar{u}-\tilde{u})^{+} \in W^{1, p(z)}(\Omega)$. We have

$$
\begin{aligned}
& \left\langle A_{p(z)}(\tilde{u}),(\bar{u}-\tilde{u})^{+}\right\rangle+\left\langle A_{q(z)}(\tilde{u}),(\bar{u}-\tilde{u})^{+}\right\rangle+\int_{\Omega} \xi(z)|\tilde{u}|^{p(z)-2} \tilde{u}(\bar{u}-\tilde{u})^{+} d z \\
= & \int_{\Omega}\left[\bar{u}^{-\eta(z)}+\lambda f(z, \bar{u})\right](\bar{u}-\tilde{u})^{+} d z \text { (see Eq. 24) } \\
\geq & \left.\int_{\Omega} \bar{u}^{-\eta(z)}(\bar{u}-\tilde{u})^{+} d z \text { (see hypothesis } H_{1}(i)\right) \\
= & \left\langle A_{p(z)}(\bar{u}),(\bar{u}-\tilde{u})^{+}\right\rangle+\left\langle A_{q(z)},(\bar{u}-\tilde{u})^{+}\right\rangle+\int_{\Omega} \xi(z) \bar{u}^{p(z)-1}(\bar{u}-\tilde{u})^{+} d z, \\
\Rightarrow & \tilde{u} .
\end{aligned}
$$

Next, in Eq. 25 we choose $h=(\tilde{u}-\hat{u})^{+} \in W^{1, p(z)}(\Omega)$. We have

$$
\begin{aligned}
& \left\langle A_{p(z)}(\tilde{u}),(\tilde{u}-\hat{u})^{+}\right\rangle+\left\langle A_{q(z)},(\tilde{u}-\hat{u})^{+}\right\rangle+\int_{\Omega} \xi(z) \tilde{u}^{p(z)-1}(\tilde{u}-\hat{u})^{+} d z \\
= & \int_{\Omega}\left[\hat{u}^{-\eta(z)}+\lambda f(z, \hat{u})\right](\tilde{u}-\hat{u})^{+} d z(\text { see Eq. 24) } \\
\leq & \int_{\Omega}\left[\hat{u}^{-\eta(z)}+1\right](\tilde{u}-\hat{u})^{+} d z\left(\text { since } \lambda \in\left(0, \lambda_{0}\right],\right. \text { see Eq. 23) } \\
\leq & \int_{\Omega}\left[\bar{u}^{-\eta(z)}+1\right](\tilde{u}-\hat{u})^{+} d z(\text { see Eq. 22) } \\
= & \left\langle A_{p(z)}(\hat{u}),(\tilde{u}-\hat{u})^{+}\right\rangle+\left\langle A_{q(z)}(\hat{u}),(\tilde{u}-\hat{u})^{+}\right\rangle+\int_{\Omega} \xi(z) \tilde{u}^{p(z)-1}(\tilde{u}-\hat{u})^{+} d z, \\
\Rightarrow & \hat{u} .
\end{aligned}
$$

So, we have proved that

$$
\tilde{u} \in[\bar{u}, \hat{u}] .
$$

From Eqs. 26, 24 and 25, we infer that

$$
\begin{aligned}
& \tilde{u} \in S_{\lambda}, \\
\Rightarrow & \left(0, \lambda_{0}\right] \subseteq \mathcal{L} \neq \emptyset,
\end{aligned}
$$

which concludes the proof.

Proposition 4.2 If hypotheses $H_{0}, H_{1}$ hold and $\lambda \in \mathcal{L}$, then $\bar{u} \leq u$ for all $u \in S_{\lambda}$.

Proof Let $u \in S_{\lambda}$ and consider the following function

$$
\hat{\mu}_{+}(z, x)= \begin{cases}x^{-\eta(z)}, & \text { if } 0 \leq x \leq u(z) \\ u(z)^{-\eta(z)}, & \text { if } u(z)<x .\end{cases}
$$

Evidently, this function is Carathéodory on $\Omega \times(\mathbb{R} \backslash\{0\})$ and is singular at $x=0$. We consider the purely singular problem

$$
\left\{\begin{array}{l}
-\Delta_{p(z)} u(z)-\Delta_{q(z)} u(z)+\xi(z) u(z)^{p(z)-1}=\hat{\mu}_{+}(z, u(z)) \text { in } \Omega, \\
\frac{\partial u}{\partial n}=0 \text { on } \partial \Omega, u>0 .
\end{array}\right.
$$


Reasoning as for problem (8), we show that problem (28) admits a positive solution $u^{*} \in \operatorname{int} C_{+}$(see also Papageorgiou et al. [36, Proposition 10]). We have

$$
\begin{aligned}
& \left\langle A_{p(z)}\left(u^{*}\right),\left(u^{*}-u\right)^{+}\right\rangle+\left\langle A_{q(z)}\left(u^{*}\right),\left(u^{*}-u\right)^{+}\right\rangle+\int_{\Omega} \xi(z)\left(u^{*}\right)^{p(z)-1}\left(u^{*}-u\right)^{+} d z \\
= & \int_{\Omega} u^{-\eta(z)}\left(u^{*}-u\right)^{+} d z \text { (see Eq. 27) } \\
& \left(\text { recall that, by definition, } u^{-\eta(z)} h \in L^{1}(\Omega) \text { for all } h \in W^{1, p(z)}(\Omega)\right) \\
\leq & \int_{\Omega}\left[u^{-\eta(z)}+\lambda f(z, u)\right]\left(u^{*}-u\right)^{+} d z \\
= & \left\langle A_{p(z)}(u),\left(u^{*}-u\right)^{+}\right\rangle+\left\langle A_{q(z)}(u),\left(u^{*}-u\right)^{+}\right\rangle \\
& +\int_{\Omega} \xi(z) u^{p(z)-1}\left(u^{*}-u\right)^{+} d z\left(\text { since } u \in S_{\lambda}\right) \\
\Rightarrow u^{*} \leq & u
\end{aligned}
$$

So, we have

$$
u^{*} \in[0, u], u^{*} \neq 0 .
$$

From Eqs. 29, 27 and Proposition 3.4, it follows that

$$
\begin{aligned}
& u^{*}=\bar{u} \\
\Rightarrow & \bar{u} \leq u \text { for all } u \in S_{\lambda} .
\end{aligned}
$$

The proof is now complete.

According to the previous proposition, if $\lambda \in \mathcal{L}$ and $u \in S_{\lambda}$, then

$$
0 \leq u^{-\eta(z)} \leq \bar{u}^{-\eta(z)} \text { with } \bar{u}(\cdot)^{-\eta(\cdot)} \in L^{\infty}(\Omega) .
$$

Then the anisotropic regularity theory (see Fan [15]) and the anisotropic maximum principle (see Zhang [41]), imply the following result concerning the solution set $S_{\lambda}$.

Proposition 4.3 If hypotheses $H_{0}, H_{1}$ hold and $\lambda \in \mathcal{L}$, then $S_{\lambda} \subseteq$ int $C_{+}$.

Let $\lambda^{*}=\sup \mathcal{L}$.

Proposition 4.4 If hypotheses $H_{0}, H_{1}$ hold, then $\lambda^{*}<\infty$.

Proof On account of hypotheses $H_{1}(i i),(i i i)$, (iv), we can find $\lambda_{0}>0$ big such that

$$
\lambda_{0} f(z, x) \geq \xi(z) x^{p(z)-1} \text { for a.a. } z \in \Omega \text {, all } x \geq 0 .
$$

Let $\lambda>\lambda_{0}$ and suppose that $\lambda \in \mathcal{L}$. Then we can find $u_{\lambda} \in S_{\lambda} \subseteq \operatorname{int} C_{+}$(see Proposition 4.3). Let $m_{\lambda}=\min _{\bar{\Omega}} u_{\lambda}>0$ (since $u_{\lambda} \in \operatorname{int} C_{+}$). Let $\delta \in(0,1], \rho=$ 
$\max \left\{\left\|u_{\lambda}\right\|_{\infty}, m_{\lambda}+1\right\}$ and let $\hat{\xi}_{\rho}>0$ be as postulated by hypothesis $H_{1}(v)$. We set $m_{\lambda}^{\delta}=m_{\lambda}+\delta$

$$
\begin{aligned}
& -\Delta_{p(z)} m_{\lambda}^{\delta}-\Delta_{q(z)} m_{\lambda}^{\delta}+\left[\xi(z)+\hat{\xi}_{\rho}\right]\left(m_{\lambda}^{\delta}\right)^{p(z)-1}-\left(m_{\lambda}^{\delta}\right)^{-\eta(z)} \\
\leq & {\left[\xi(z)+\hat{\xi}_{\rho}\right] m_{\lambda}^{p(z)-1}+\chi(\delta) \text { with } \chi(\delta) \rightarrow 0^{+} \text {as } \delta \rightarrow 0^{+} } \\
\leq & \lambda_{0} f\left(z, m_{\lambda}\right)+\hat{\xi}_{\rho} m_{\lambda}^{p(z)-1}+\chi(\delta) \text { (see Eq. 30) } \\
= & \lambda f\left(z, m_{\lambda}\right)+\hat{\xi}_{\rho} m_{\lambda}^{p(z)-1}-\left[\lambda-\lambda_{0}\right] f\left(z, m_{\lambda}\right)+\chi(\delta) \\
\leq & \lambda f\left(z, m_{\lambda}\right)+\hat{\xi}_{\rho} m_{\lambda}^{p(z)-1}-\left[\lambda-\lambda_{0}\right] \hat{\mu}_{\lambda}+\chi(\delta) \\
& \text { with } \hat{\mu}_{\lambda}>0\left(\text { see hypothesis } H_{1}(i v)\right) \\
\leq & \left.\lambda f\left(z, u_{\lambda}\right)+\hat{\xi}_{\rho} u_{\lambda}^{p(z)-1} \text { for } \delta>0 \text { small (see hypothesis } H_{1}(v)\right) \\
= & -\Delta_{p(z)} u_{\lambda}-\Delta_{q(z)} u_{\lambda}+\left[\xi(z)+\hat{\xi}_{\rho}\right] u_{\lambda}^{p(z)-1}-u_{\lambda}^{-\eta(z)}\left(\text { since } u_{\lambda} \in S_{\lambda}\right), \\
\Rightarrow & u_{\lambda}-m_{\lambda}^{\delta} \in D_{+} \text {for } \delta>0 \text { small (see Proposition 2.3), }
\end{aligned}
$$

a contradiction to the definition of $m_{\lambda}>0$.

So, we have $\lambda^{*} \leq \lambda_{0}<\infty$.

Next, we show that $\mathcal{L}$ is, in fact, an interval.

Proposition 4.5 If hypotheses $H_{0}, H_{1}$ hold, $\lambda \in \mathcal{L}$ and $0<\mu<\lambda$, then $\mu \in \mathcal{L}$.

Proof Since $\lambda \in \mathcal{L}$, we can find $u_{\lambda} \in S_{\lambda} \subseteq$ int $C_{+}$(see Proposition 4.3). Then we have

$$
\begin{aligned}
& -\Delta_{p(z)} u_{\lambda}-\Delta_{q(z)} u_{\lambda}+\xi(z) u_{\lambda}^{p(z)-1} \\
= & u_{\lambda}^{-\eta(z)}+\lambda f\left(z, u_{\lambda}\right) \\
\geq & u_{\lambda}^{-\eta(z)}+\mu f\left(z, u_{\lambda}\right) \text { in } \Omega \\
& \quad\left(\text { recall that } 0<\mu<\lambda \text { and see hypothesis } H_{1}(i)\right) .
\end{aligned}
$$

Also we have

$$
\begin{aligned}
& -\Delta_{p(z)} \bar{u}-\Delta_{q(z)} \bar{u}+\xi(z) \bar{u}^{p(z)-1} \\
= & \bar{u}^{-\eta(z)} \\
\leq & \left.\bar{u}^{-\eta(z)}+\mu f(z, \bar{u}) \text { in } \Omega \text { (see hypothesis } H_{1}(i)\right) .
\end{aligned}
$$

From Proposition 4.2 we know that $\bar{u} \leq u_{\lambda}$. So, we can define the following truncation of the reaction of problem $\left(P_{\mu}\right)$

$$
\hat{\tau}_{\mu}(z, x)= \begin{cases}\bar{u}^{-\eta(z)}+\mu f(z, \bar{u}(z)), & \text { if } x<\bar{u}(z) \\ x^{-\eta(z)}+\mu f(z, x), & \text { if } \bar{u}(z) \leq x \leq u_{\lambda}(z) \\ u_{\lambda}(z)^{-\eta(z)}+\mu f\left(z, u_{\lambda}(z)\right), & \text { if } u_{\lambda}(z)<x\end{cases}
$$

This is a Carathéodory function. We set $\hat{T}_{\mu}(z, x)=\int_{0}^{x} \hat{\tau}_{\mu}(z, s) d s$ and consider the $C^{1}$-functional $\hat{w}_{\mu}: W^{1, p(z)}(\Omega) \rightarrow \mathbb{R}$ defined by

$$
\hat{w}_{\mu}(u)=\gamma_{p(z)}(u)+\int_{\Omega} \frac{1}{q(z)}|D u|^{q(z)} d z-\int_{\Omega} \hat{T}_{\mu}(z, u) d z \text { for all } u \in W^{1, p(z)}(\Omega) .
$$


As before (see the proof of Proposition 4.1), using the direct method of the calculus of variations and Eqs. 31, 32, we can find $u_{\mu} \in W^{1, p(z)}(\Omega)$ such that

$$
\begin{aligned}
& u_{\mu} \in K_{w_{\lambda}} \subseteq\left[\bar{u}, u_{\lambda}\right] \cap \operatorname{int} C_{+}, \\
\Rightarrow & \left.u_{\mu} \in S_{\mu} \text { (see Eq. } 33\right), \\
\Rightarrow & \mu \in \mathcal{L} .
\end{aligned}
$$

The proof is now complete.

Remark 4.6 As a byproduct of this proof we have that if $0<\mu<\lambda \in \mathcal{L}$ and $u_{\lambda} \in S_{\lambda} \subseteq$ int $C_{+}$then we can find $u_{\mu} \in S_{\mu} \subseteq$ int $C_{+}$such that

$$
u_{\lambda}-u_{\mu} \in C_{+} \backslash\{0\} .
$$

In fact we can improve this result using the strong comparison principle (see Proposition 2.3).

Proposition 4.7 If hypotheses $H_{0}, H_{1}$ hold, $0<\mu<\lambda \in \mathcal{L}$ and $u_{\lambda} \in S_{\lambda} \subseteq$ int $C_{+}$, then $\mu \in \mathcal{L}$ and there exists $u_{\mu} \in S_{\mu} \subseteq$ int $C_{+}$such that $u_{\lambda}-u_{\mu} \in D_{+}$.

Proof From Proposition 4.5 and its proof, we already know that $\mu \in \mathcal{L}$ and we can find $u_{\mu} \in S_{\mu} \subseteq$ int $C_{+}$such that

$$
u_{\mu} \leq u_{\lambda}, u_{\mu} \neq u_{\lambda}
$$

Let $\rho=\left\|u_{\lambda}\right\|_{\infty}$ and let $\hat{\xi}_{\rho}>0$ be as postulated by hypothesis $H_{1}(v)$. We have

$$
\begin{aligned}
& -\Delta_{p(z)} u_{\mu}-\Delta_{q(z)} u_{\mu}+\left[\xi(z)+\hat{\xi}_{\rho}\right] u_{\mu}^{p(z)-1}-u_{\mu}^{-\eta(z)} \\
= & \mu f\left(z, u_{\mu}\right)+\hat{\xi}_{\rho} u_{\mu}^{p(z)-1} \\
= & \lambda f\left(z, u_{\mu}\right)+\hat{\xi}_{\rho} u_{\mu}^{p(z)-1}-(\lambda-\mu) f\left(z, u_{\mu}\right) \\
\leq & \left.\lambda f\left(z, u_{\lambda}\right)+\hat{\xi}_{\rho} u_{\lambda}^{p(z)-1} \text { (see Eq. } 34 \text { and hypothesis } H_{1}(v)\right) \\
= & -\Delta_{p(z)} u_{\lambda}-\Delta_{q(z)} u_{\lambda}+\left[\xi(z)+\hat{\xi}_{\rho}\right] u_{\lambda}^{p(z)-1} \text { in } \Omega .
\end{aligned}
$$

On account of hypothesis $H_{1}(i v)$ and since $u_{\mu} \in \operatorname{int} C_{+}$, we have

$$
0<\hat{\mu}_{\mu} \leq(\lambda-\mu) f\left(z, u_{\mu}(z)\right) \text { for a.a. } z \in \Omega \text {. }
$$

Hence from Eq. 35 and Proposition 2.3, it follows that

$$
u_{\lambda}-u_{\mu} \in D_{+} \text {. }
$$

The proof is now complete.

Proposition 4.8 If hypotheses $H_{0}, H_{1}$ hold and $\lambda \in\left(0, \lambda^{*}\right)$, then problem $\left(P_{\lambda}\right)$ admits at least two positive solutions

$$
u_{0}, \hat{u} \in \operatorname{int} C_{+}, u_{0} \leq \hat{u}, u_{0} \neq \hat{u} .
$$

Proof Let $0<\lambda<\vartheta<\lambda^{*}$. We know that $\vartheta \in \mathcal{L}$ (see Proposition 4.5). Moreover, according to Proposition 4.7, we can find $u_{\vartheta} \in S_{\vartheta} \subseteq$ int $C_{+}$and $u_{0} \in S_{\lambda} \subseteq$ int $C_{+}$such that

$$
u_{\vartheta}-u_{0} \in \operatorname{int} C_{+} \text {. }
$$

We introduce the Carathéodory function $d_{\lambda}(z, x)$ defined by

$$
d_{\lambda}(z, x)= \begin{cases}u_{0}(z)^{-\eta(z)}+\lambda f\left(z, u_{0}(z)\right), & \text { if } x \leq u_{0}(z) \\ x^{-\eta(z)}+\lambda f(z, x), & \text { if } u_{0}(z)<x .\end{cases}
$$


Also we consider the following truncation of $d_{\lambda}(z, \cdot)$ :

$$
\hat{d}_{\lambda}(z, x)= \begin{cases}d_{\lambda}(z, x), & \text { if } x \leq u_{\vartheta}(z) \\ d_{\lambda}\left(z, u_{\vartheta}(z)\right), & \text { if } u_{\vartheta}(z)<x .\end{cases}
$$

This is also a Carathéodory function. We set

$$
D_{\lambda}(z, x)=\int_{0}^{x} d_{\lambda}(z, s) d s \text { and } \hat{D}_{\lambda}(z, x)=\int_{0}^{x} \hat{d}_{\lambda}(z, s) d s .
$$

Then we consider the $C^{1}$-functionals $v_{\lambda}, \hat{v}_{\lambda}: W^{1, p(z)}(\Omega) \rightarrow \mathbb{R}$ defined by

$$
\begin{aligned}
& v_{\lambda}(u)=\gamma_{p(z)}(u)+\int_{\Omega} \frac{1}{q(z)}|D u|^{q(z)}-\int_{\Omega} D_{\lambda}(z, u) d z, \\
& \hat{v}_{\lambda}(u)=\gamma_{p(z)}(u)+\int_{\Omega} \frac{1}{q(z)}|D u|^{q(z)} d z-\int_{\Omega} \hat{D}_{\lambda}(z, u) d z \text { for all } u \in W^{1, p(z)}(\Omega) .
\end{aligned}
$$

From Eqs. 37, 38 and the anisotropic regularity theory, we have

$$
\begin{gathered}
K_{v_{\lambda}} \subseteq\left[u_{0}\right) \cap \operatorname{int} C_{+}, \\
K_{\hat{v}_{\lambda}} \subseteq\left[u_{0}, u_{\vartheta}\right] \cap \operatorname{int} C_{+} .
\end{gathered}
$$

On account of Eqs. 37 and 39, we see that we may assume that

$$
K_{v_{\lambda}} \cap\left[u_{0}, u_{\vartheta}\right]=\left\{u_{0}\right\} .
$$

Otherwise we already have a second positive smooth solution bigger that $u_{0}$ (see Eqs. 37, 39). Also from Eqs. 37 and 38 we see that

$$
\left.v_{\lambda}\right|_{\left[0, u_{\vartheta}\right]}=\left.\hat{v}_{\lambda}\right|_{\left[0, u_{\vartheta}\right]},\left.v_{\lambda}^{\prime}\right|_{\left[0, u_{\vartheta}\right]}=\left.\hat{v}_{\lambda}^{\prime}\right|_{\left[0, u_{\vartheta}\right]} .
$$

The functional $\hat{v}_{\lambda}(\cdot)$ is coercive and sequentially weakly lower semicontinuous. So, we can find $\tilde{u}_{0} \in W^{1, p(z)}(\Omega)$ such that

$$
\begin{aligned}
& \hat{v}_{\lambda}\left(\tilde{u}_{0}\right)=\min \left\{\hat{v}_{\lambda}(u): u \in W^{1, p(z)}(\Omega)\right\}, \\
\Rightarrow & \tilde{u}_{0} \in K_{\hat{v}_{\lambda}} \subseteq\left[u_{0}, u_{\vartheta}\right] \cap \operatorname{int} C_{+}(\text {see Eq. } 40), \\
\Rightarrow & \tilde{u}_{0}=u_{0}(\text { see Eqs. } 41,42), \\
\Rightarrow & u_{0} \text { is a local } C^{1}(\bar{\Omega}) \text {-minimizer of } v_{\lambda}(\cdot)(\text { see Eqs. } 36,42), \\
\Rightarrow & u_{0} \text { is a local } W^{1, p(z)}(\Omega) \text {-minimizer of } v_{\lambda}(\cdot) \\
& (\text { see Gasinski and Papageorgiou }[16, \text { Proposition 3.3]). }
\end{aligned}
$$

From Eqs. 39 and 37 it is clear that we may assume that

$$
K_{v_{\lambda}} \text { is finite. }
$$

Indeed, otherwise we already have a whole sequence of positive smooth solutions all bigger than $u_{0}$ and so we are done.

From Eqs. 43, 44 and Theorem 5.7.6 of Papageorgiou et al. [32, p. 449], we know that we can find $\rho \in(0,1)$ small such that

$$
v_{\lambda}\left(u_{0}\right)<\inf \left\{v_{\lambda}(u):\left\|u-u_{0}\right\|=\rho\right\}=m_{\lambda} .
$$

Also hypothesis $H_{1}(\mathrm{ii})$ and Eq. 37 imply that if $u \in \operatorname{int} C_{+}$, then

$$
v_{\lambda}(t u) \rightarrow-\infty \text { as } t \rightarrow+\infty \text {. }
$$

Claim: $v_{\lambda}(\cdot)$ satisfies the $C$-condition. 
We consider a sequence $\left\{u_{n}\right\}_{n \geq 1} \subseteq W^{1, p(z)}(\Omega)$ such that

$$
\begin{gathered}
\left|v_{\lambda}\left(u_{n}\right)\right| \leq C_{7} \text { for some } C_{7}>0, \text { all } n \in \mathbb{N}, \\
\left(1+\left\|u_{n}\right\|\right) v_{\lambda}^{\prime}\left(u_{n}\right) \rightarrow 0 \text { in } W^{1, p(z)}(\Omega)^{*} \text { as } n \rightarrow \infty .
\end{gathered}
$$

From Eq. 48 we have

$$
\left.\left|\left\langle A_{p(z)}\left(u_{n}\right), h\right\rangle+\left\langle A_{q(z)}\left(u_{n}\right), h\right\rangle+\int_{\Omega} \xi(z)\right| u_{n}\right|^{p(z)-2} u_{n} h d z-\int_{\Omega} d_{\lambda}\left(z, u_{n}\right) h d z \mid \leq \frac{\varepsilon_{n}\|h\|}{1+\left\|u_{n}\right\|}
$$

for all $h \in W^{1, p(z)}(\Omega)$, with $\varepsilon_{n} \rightarrow 0^{+}$.

In Eq. 49, we choose $h=-u_{n}^{-} \in W^{1, p(z)}(\Omega)$ and obtain

$$
\begin{aligned}
& \rho_{p(z)}\left(D u_{n}^{-}\right)+\rho_{q(z)}\left(D u_{n}^{-}\right)+\int_{\Omega} \xi(z)\left(u_{n}^{-}\right)^{p(z)} d z \leq C_{8} \\
& \text { for some } C_{8}>0, \text { all } n \in \mathbb{N} \text { (see Eq. 37), } \\
\Rightarrow & \left\{u_{n}^{-}\right\}_{n \geq 1} \subseteq W^{1, p(z)}(\Omega) \text { is bounded. }
\end{aligned}
$$

Next, we choose in Eq. $49 h=u_{n}^{+} \in W^{1, p(z)}(\Omega)$ and we obtain

$$
-\rho_{p(z)}\left(D u_{n}^{+}\right)-\rho\left(D u_{n}^{+}\right)-\int_{\Omega} \xi(z)\left(u_{n}^{+}\right)^{p(z)} d z+\int_{\Omega} d_{\lambda}\left(z, u_{n}^{+}\right) u_{n}^{+} d z \leq \varepsilon_{n}
$$

for all $n \in \mathbb{N}$.

From Eqs. 47 and 50, we have

$$
\rho_{p(z)}\left(D u_{n}^{+}\right)+\rho_{q(z)}\left(D u_{n}^{+}\right)+\int_{\Omega} \xi(z)\left(u_{n}^{+}\right)^{p(z)} d z-\int_{\Omega} p_{+} D_{\lambda}\left(z, u_{n}^{+}\right) d z \leq C_{9}
$$

for some $C_{9}>0$, all $n \in \mathbb{N}$ (recall $\left.q_{-}<p_{-}\right)$.

We add Eqs. 51 and 52. Then

$$
\int_{\Omega} I_{\lambda}\left(z, u_{n}^{+}\right) d z \leq C_{10}
$$

for some $C_{10}>0$, all $n \in \mathbb{N}$ (see Eq. 37).

Suppose that $\left\{u_{n}^{+}\right\}_{n \geq 1} \subseteq W^{1, p(z)}(\Omega)$ is not bounded. So, we may assume that

$$
\left\|u_{n}^{+}\right\| \rightarrow+\infty \text { as } n \rightarrow \infty \text {. }
$$

We set $y_{n}=\frac{u_{n}^{+}}{\left\|u_{n}^{+}\right\|}$for all $n \in \mathbb{N}$. Then $\left\|y_{n}\right\|=1, y_{n} \geq 0$ for all $n \in \mathbb{N}$. So, we may assume that

$$
y_{n} \stackrel{w}{\rightarrow} y \text { in } W^{1, p(z)}(\Omega) \text { and } y_{n} \rightarrow y \text { in } L^{p(z)}(\Omega), y \geq 0 .
$$

Initially we assume that $y \not \equiv 0$. Let $\hat{\Omega}=\{z \in \Omega: y(z)>0\}$. From Eq. 55 we see that $|\hat{\Omega}|_{N}>0\left(\right.$ by $|\cdot|_{N}$ we denote the Lebesgue measure on $\left.\mathbb{R}^{N}\right)$ and

$$
u_{n}^{+}(z) \rightarrow+\infty \text { for a.a. } z \in \hat{\Omega} .
$$

Hypothesis $H_{1}(\mathrm{ii})$ implies

$$
\begin{aligned}
& \frac{F\left(z, u_{n}^{+}(z)\right)}{\left\|u_{n}^{+}\right\|^{p_{+}}}=\frac{F\left(z, u_{n}^{+}(z)\right)}{u_{n}^{+}(z)^{p_{+}}} y_{n}(z)^{p_{+}} \rightarrow+\infty \text { for a.a. } z \in \hat{\Omega}, \\
\Rightarrow & \int_{\hat{\Omega}} \frac{F\left(z, u_{n}^{+}\right)}{\left\|u_{n}^{+}\right\|^{p_{+}}} d z \rightarrow+\infty \text { (by Fatou's lemma), } \\
\Rightarrow & \left.\int_{\Omega} \frac{F\left(z, u_{n}^{+}\right)}{\left\|u_{n}^{+}\right\|^{p_{+}}} d z \rightarrow+\infty \text { (since } F \geq 0, \text { see hypothesis } H_{1}(i)\right) .
\end{aligned}
$$


From Eqs. 47 and 50, we have

$$
\begin{aligned}
& \quad-\gamma_{p(z)}\left(u_{n}^{+}\right)-\int_{\Omega} \frac{1}{q(z)}\left|D u_{n}^{+}\right|^{q(z)} d z-\int_{\Omega} \frac{\xi(z)}{p(z)}\left(u_{n}^{+}\right)^{p(z)} d z+\int_{\Omega} D_{\lambda}\left(z, u_{n}^{+}\right) d z \leq C_{11} \\
& \quad \text { for some } C_{11}>0, \text { all } n \in \mathbb{N}, \\
& \Rightarrow \int_{\Omega} \frac{\lambda F\left(z, u_{n}^{+}\right)}{\left\|u_{n}^{+}\right\|^{p_{+}}} d z \leq C_{12} \\
& \quad \text { for some } C_{12}>0, \text { all } n \in \mathbb{N} \text { (see Eqs. } 54 \text { and 37). }
\end{aligned}
$$

Comparing Eqs. 56 and 57, we have a contradiction.

Now we assume that $y \equiv 0$. We consider the $C^{1}$-functional $\tilde{v}_{\lambda}: W^{1, p(z)}(\Omega) \rightarrow \mathbb{R}$ defined by

$$
\tilde{v}_{\lambda}(u)=\frac{1}{p_{+}}\left[\int_{\Omega}|D u|^{p(z)} d z+\int_{\Omega} \xi(z)|u|^{p(z)} d z\right]-\int_{\Omega} D_{\lambda}(z, u) d z
$$

for all $u \in W^{1, p(z)}(\Omega)$.

Evidently we have

$$
\tilde{v}_{\lambda} \leq v_{\lambda}
$$

Let $k_{n}(t)=\tilde{v}_{\lambda}\left(t u_{n}^{+}\right)$for all $t \in[0,1]$, all $n \in \mathbb{N}$. We can find $t_{n} \in[0,1]$ such that

$$
k_{n}\left(t_{n}\right)=\max _{0 \leq t \leq 1} k_{n}(t)
$$

For $\hat{\eta} \geq 1$ let $w_{n}=(2 \hat{\eta})^{\frac{1}{p_{-}}} y_{n} n \in \mathbb{N}$. Then

$$
\begin{aligned}
& \left.w \rightarrow 0 \text { in } L^{p(z)}(\Omega) \text { (see Eq. } 55 \text { and recall that } y \equiv 0\right) \\
\Rightarrow & \int_{\Omega} D_{\lambda}\left(z, w_{n}\right) d z \rightarrow 0 \text { as } n \rightarrow \infty
\end{aligned}
$$

On account of Eq. 54, we see that we can find $n_{0} \in \mathbb{N}$ such that

$$
(2 \hat{\eta})^{\frac{1}{p_{-}}} \frac{1}{\left\|u_{n}^{+}\right\|} \leq 1 \text { for all } n \geq n_{0} .
$$

From Eqs. 59 and 61, we have

$$
\begin{aligned}
& k_{n}\left(t_{n}\right) \geq k_{n}\left(\frac{(2 \hat{\eta})^{1 / p_{-}}}{\left\|u_{n}^{+}\right\|}\right), \\
\Rightarrow \tilde{v}_{\lambda}\left(t_{n} u_{n}^{+}\right) \geq & \tilde{v}_{\lambda}\left((2 \hat{\eta})^{1 / p_{-}} y_{n}\right)=\tilde{v}_{\lambda}\left(w_{n}\right), \\
\Rightarrow \tilde{v}_{\lambda}\left(t_{n} u_{n}^{+}\right) \geq & \frac{2 \hat{\eta}}{p_{+}}\left[\rho_{p(z)}\left(D y_{n}\right)+\rho_{p(z), \xi}\left(y_{n}\right)\right]-\int_{\Omega} D_{\lambda}\left(z, w_{n}\right) d z \\
\geq & \frac{2 \hat{\eta}}{p_{+}} C_{13}\left\|y_{n}\right\|-\int_{\Omega} D_{\lambda}\left(z, w_{n}\right) d z \text { for some } C_{13}>0 \\
\geq & \frac{\hat{\eta}}{p_{+}} C_{13} \text { for all } n \geq n_{1} \geq n_{0} \\
& \text { (see Eq. } \left.60 \text { and recall that }\left\|y_{n}\right\|=1\right) .
\end{aligned}
$$

But $\hat{\eta} \geq 1$ is arbitrary. So, it follows that

$$
\tilde{v}_{\lambda}\left(t_{n} u_{n}^{+}\right) \rightarrow+\infty \text { as } n \rightarrow \infty .
$$

We have

$$
0 \leq t_{n} u_{n}^{+} \leq u_{n}^{+} \text {for all } n \in \mathbb{N} \text {. }
$$


Hence hypothesis $H_{1}(\mathrm{iii})$ implies that

$$
\int_{\Omega} I_{\lambda}\left(z, t_{n} u_{n}^{+}\right) d z \leq \int_{\Omega} I_{\lambda}\left(z, u_{n}^{+}\right) d z+\left\|\hat{\vartheta}_{\lambda}\right\|_{1} \leq C_{14}
$$

for some $C_{14}>0$, all $n \in \mathbb{N}$ (see Eq. 53).

We know that

$$
\tilde{v}_{\lambda}(0)=0 \text { and } \tilde{v}_{\lambda}\left(u_{n}\right) \leq C_{7} \text { for all } n \in \mathbb{N} \text { (see Eqs. } 47 \text { and 58). }
$$

Then from Eq. 62 it follows that

$$
t_{n} \in(0,1) \text { for all } n \geq n_{2} .
$$

So, from Eq. 59 we have

$$
\begin{aligned}
0= & \left.t_{n} \frac{d}{d t} \tilde{v}_{\lambda}\left(t u_{n}^{+}\right)\right|_{t=t_{n}} \\
= & \left\langle\tilde{v}_{\lambda}^{\prime}\left(t_{n} u_{n}^{+}\right), t_{n} u_{n}^{+}\right\rangle \text {for all } n \geq n_{2} \text { (by the chain rule), } \\
\Rightarrow \quad & \rho_{p(z)}\left(D\left(t_{n} u_{n}^{+}\right)\right)+\rho_{p(z), \xi}\left(t_{n} u_{n}^{+}\right)=\int_{\Omega} d_{\lambda}\left(z, t_{n} u_{n}^{+}\right)\left(t_{n} u_{n}^{+}\right) d z \text { for all } n \geq n_{2}, \\
\Rightarrow \quad & \rho_{p(z)}\left(D\left(t_{n} u_{n}^{+}\right)\right)+\rho_{p(z), \xi}\left(t_{n} u_{n}^{+}\right)-\int_{\Omega} p_{+} D_{\lambda}\left(z, t_{n} u_{n}^{+}\right) d z \leq C_{15} \\
& \quad \text { for some } C_{15}>0, \text { all } n \geq n_{2}(\text { see Eqs. } 63 \text { and 37), } \\
\Rightarrow \quad & p_{+} \tilde{v}_{\lambda}\left(t_{n} u_{n}^{+}\right) \leq C_{15} \text { for all } n \geq n_{2} .
\end{aligned}
$$

Comparing Eqs. 62 and 64 we have a contradiction.

Therefore we infer that

$$
\begin{aligned}
& \left\{u_{n}^{+}\right\}_{n \geq 1} \subseteq W^{1, p(z)}(\Omega) \text { is bounded, } \\
\Rightarrow & \left\{u_{n}\right\}_{n \geq 1} \subseteq W^{1, p(z)}(\Omega) \text { is bounded (see Eq. 50). }
\end{aligned}
$$

So, we may assume that

$$
u_{n} \stackrel{w}{\rightarrow} u \text { in } W^{1, p(z)}(\Omega) \text { and } u_{n} \rightarrow u \text { in } L^{r(z)}(\Omega) \text { as } n \rightarrow \infty .
$$

In Eq. 49 we choose $h=u_{n}-u \in W^{1, p(z)}(\Omega)$, pass to the limit as $n \rightarrow \infty$ and use Eq. 63. Then reasoning as in the proof of Proposition 3.2, using Proposition 2.2, we obtain that

$$
\begin{aligned}
& u_{n} \rightarrow u \text { in } W^{1, p(z)}(\Omega), \\
\Rightarrow & v_{\lambda}(\cdot) \text { satisfies the } C \text {-condition. }
\end{aligned}
$$

This proves the Claim.

On account of Eqs. 45, 46 and the Claim, we can apply the mountain pass theorem and find $\hat{u} \in W^{1, p(z)}(\Omega)$ such that

$$
\hat{u} \in K_{v_{\lambda}} \subseteq\left[u_{0}\right) \cap \operatorname{int} C_{+} \text {and } m_{\lambda} \leq v_{\lambda}(\hat{u}) .
$$

From Eqs. 66, 45 and 37, we obtain

$$
\hat{u} \in S_{\lambda} \subseteq \operatorname{int} C_{+}, u_{0} \leq \hat{u}, u_{0} \neq \hat{u},
$$

which concludes the proof. 
We introduce the Carathéodory function $\hat{\beta}_{\lambda}(z, x)$ defined by

$$
\hat{\beta}_{\lambda}(z, x)= \begin{cases}\bar{u}(z)^{-\eta(z)}+\lambda f(z, \bar{u}(z)), & \text { if } x \leq \bar{u}(z) \\ x^{-\eta(z)}+\lambda f(z, x), & \text { if } \bar{u}(z)<x\end{cases}
$$

We set $\hat{B}_{\lambda}(z, x)=\int_{0}^{x} \hat{\beta}(z, s) d s$ and consider the $C^{1}$-functional $\hat{\varphi}_{\lambda}: W^{1, p(z)}(\Omega) \rightarrow \mathbb{R}$ defined by

$$
\hat{\varphi}_{\lambda}(u)=\gamma_{p(z)}(u)+\int_{\Omega} \frac{1}{q(z)}|D u|^{q(z)} d z-\int_{\Omega} \hat{B}_{\lambda}(z, u) d z
$$

for all $u \in W^{1, p(z)}(\Omega)$.

Using this functional, we can establish the admissibility of the critical parameter value $\lambda^{*}>0$.

Proposition 4.9 If hypotheses $H_{0}, H_{1}$ hold, then $\lambda^{*} \in \mathcal{L}$.

Proof Let $\left\{\lambda_{n}\right\}_{n \geq 1} \subseteq\left(0, \lambda^{*}\right) \subseteq \mathcal{L}$ and assume that $\lambda_{n} \rightarrow \lambda^{*}$. We can find $u_{n} \in S_{\lambda} \subseteq$ int $C_{+}$ $n \in \mathbb{N}$ such that

$$
\hat{\varphi}_{\lambda_{n}}\left(u_{n}\right) \leq \hat{\varphi}_{\lambda_{n}}(\bar{u})<0 \text { for all } n \in \mathbb{N}
$$

(see the proof of Proposition 4.5 and Eq. 67).

Also we have

$$
\hat{\varphi}_{\lambda_{n}}^{\prime}\left(u_{n}\right)=0 \text { for all } n \in \mathbb{N} \text {. }
$$

Using Eqs. 68, 69 and reasoning as in the Claim in the proof of Proposition 4.8, we obtain

$$
\begin{aligned}
& u_{n} \rightarrow u^{*} \text { in } W^{1, p(z)}(\Omega), \bar{u} \leq u^{*}(\text { see Proposition } 4.2) \\
\Rightarrow & u^{*} \in S_{\lambda^{*}} \subseteq \operatorname{int} C_{+} \text {and so } \lambda^{*} \in \mathcal{L} .
\end{aligned}
$$

The proof is now complete.

According to the above proposition, we have

$$
\mathcal{L}=\left(0, \lambda^{*}\right]
$$

\section{Minimal Positive Solutions}

Recall that a set $S \subseteq W^{1, p(z)}(\Omega)$ is said to be downward directed, if for all $u_{1}, u_{2} \in S$, we can find $u \in S$ such that $u \leq u_{1}, u \leq u_{2}$.

As in the proof of Proposition 18 of Papageorgiou et al. [36], we prove that for every $\lambda \in \mathcal{L}$, the solution set $S_{\lambda} \subseteq$ int $C_{+}$is downward directed. We will show that $S_{\lambda}$ has a minimal element.

Proposition 5.1 If hypotheses $H_{0}, H_{1}$ hold and $\lambda \in \mathcal{L}=\left(0, \lambda^{*}\right]$, then problem $\left(P_{\lambda}\right)$ has a smallest positive solution $u_{\lambda}^{*} \in S_{\lambda} \subseteq$ int $C_{+}$(that is, $u_{\lambda}^{*} \leq u$ for all $u \in S_{\lambda}$ ).

Proof On account of Lemma 3.10 of $\mathrm{Hu}$ and Papageorgiou [19, p. 176], we can find $\left\{u_{n}\right\}_{n \geq 1} \subseteq S_{\lambda}$ decreasing (since $S_{\lambda}$ is downward directed) such that

$$
\inf _{n \geq 1} u_{n}=\inf S_{\lambda}
$$


We have

$$
\begin{aligned}
& \hat{\varphi}_{\lambda}^{\prime}\left(u_{n}\right)=0 \text { for all } n \in \mathbb{N}, \\
& \bar{u} \leq u_{n} \leq u_{1} \text { for all } n \in \mathbb{N} .
\end{aligned}
$$

From Eqs. 70 and 71, it follows that

$$
\left\{u_{n}\right\}_{n \geq 1} \subseteq W^{1, p(z)}(\Omega) \text { is bounded. }
$$

Then as in the proof of Proposition 4.5 and using the fact that $\left\{u_{n}\right\}_{n \geq 1}$ is decreasing, we obtain

$$
\begin{aligned}
& u_{n} \rightarrow u_{\lambda}^{*} \text { in } W^{1, p(z)}(\Omega), \\
\Rightarrow & u_{\lambda}^{*} \in S_{\lambda} \subseteq \operatorname{int} C_{+}, u_{\lambda}^{*}=\inf S_{\lambda},
\end{aligned}
$$

which concludes the proof.

Next we examine the map $\lambda \mapsto u_{\lambda}^{*}$ from $\mathcal{L}=\left(0, \lambda^{*}\right]$ into $C^{1}(\bar{\Omega})$.

Proposition 5.2 If hypotheses $H_{0}, H_{1}$ hold, then

(a) the map $\lambda \mapsto u_{\lambda}^{*}$ from $\mathcal{L}=\left(0, \lambda^{*}\right]$ into $C^{1}(\bar{\Omega})$ is strictly increasing in the sense that

$$
0<\mu<\lambda \leq \lambda^{*} \Rightarrow u_{\lambda}^{*}-u_{\mu}^{*} \in D_{+}
$$

(b) $\lambda \mapsto u_{\lambda}^{*}$ is left continuous from $\mathcal{L}$ into $C^{1}(\bar{\Omega})$.

Proof (a) Let $0<\mu<\lambda \leq \lambda^{*}$. On account of Proposition 4.7, we can find $u_{\mu} \in S_{\mu} \subseteq$ int $C_{+}$such that

$$
\begin{aligned}
& u_{\lambda}^{*}-u_{\mu} \in D_{+}, \\
\Rightarrow & u_{\lambda}^{*}-u_{\mu}^{*} \in D_{+} .
\end{aligned}
$$

(b) Let $\lambda_{n} \rightarrow \lambda^{-}, \lambda_{n} \in \mathcal{L}$ for all $n \in \mathbb{N}$. From Proposition 4.2 and part (a) of this proposition, we have

$$
\bar{u} \leq u_{\lambda}^{*} \leq u_{\lambda_{1}}^{*} \text { for all } n \in \mathbb{N} \text {. }
$$

It follows that

$$
\left\{u_{n}^{*}=u_{\lambda_{n}}^{*}\right\}_{n \geq 1} \subseteq W^{1, p(z)}(\Omega) \text { is bounded. }
$$

Then Proposition 3.1 of Gasinski and Papageorgiou [16] implies that

$$
\left\|u_{n}^{*}\right\|_{\infty} \leq C_{16} \text { for some } C_{16}>0 \text {, all } n \in \mathbb{N} \text {. }
$$

Using Theorem 1.3 of Fan [15] (see also Lieberman [21]), we see that we can find $\alpha \in$ $(0,1)$ and $C_{17}>0$ such that

$$
u_{n}^{*} \in C^{1, \alpha}(\bar{\Omega}) \text { and }\left\|u_{n}^{*}\right\|_{C^{1, \alpha}(\bar{\Omega})} \leq C_{17} \text { for all } n \in \mathbb{N} .
$$

From Eq. 72, the compact embedding of $C^{1, \alpha}(\bar{\Omega})$ into $C^{1}(\bar{\Omega})$ and the monotonicity of the sequence $\left\{u_{n}^{*}\right\}_{n \geq 1}$, we have that

$$
u_{n}^{*} \rightarrow \hat{u}_{\lambda}^{*} \text { in } C^{1}(\bar{\Omega}) .
$$

If $\hat{u}_{\lambda}^{*} \neq u_{\lambda}^{*}$, then there exists $z_{0} \in \bar{\Omega}$ such that

$$
\begin{aligned}
u_{\lambda}^{*}\left(z_{0}\right) & <\hat{u}_{\lambda}^{*}\left(z_{0}\right), \\
\Rightarrow & u_{\lambda}^{*}\left(z_{0}\right)<u_{n}^{*}\left(z_{0}\right) \text { for all } n \geq n_{0} \text { (see Eq. 73), }
\end{aligned}
$$

contradicting part $(a)$. So $\hat{u}_{\lambda}^{*}=u_{\lambda}^{*}$ and we have the left continuity of the map $\lambda \mapsto u_{\lambda}^{*}$. 
The next theorem summarizes our main contributions in this paper concerning problem $\left(P_{\lambda}\right)$.

\section{Theorem 5.3 If hypotheses $H_{0}, H_{1}$ hold, then there exists $\lambda^{*}<\infty$ such that}

(a) for all $\lambda \in\left(0, \lambda^{*}\right)$, problem $\left(P_{\lambda}\right)$ has at least two positive solutions $u_{0}, \hat{u} \in$ int $C_{+}$, $u_{0} \leq \hat{u}, u_{0} \neq \hat{u}$;

(b) for $\lambda=\lambda^{*}$, problem $\left(P_{\lambda}\right)$ has at least one positive solution $u^{*} \in \operatorname{int} C_{+}$;

(c) for all $\lambda>\lambda^{*}$, problem $\left(P_{\lambda}\right)$ has no positive solutions;

(d) for all $\lambda \in \mathcal{L}=\left(0, \lambda^{*}\right]$, problem $\left(P_{\lambda}\right)$ has a smallest positive solution $u_{\lambda}^{*} \in$ int $C_{+}$ and the map $\lambda \mapsto u_{\lambda}^{*}$ is strictly increasing and left-continuous from $\mathcal{L}$ into $C^{1}(\bar{\Omega})$.

Acknowledgments The authors wish to thank the two anonymous reviewers for their remarks and constructive criticisms that helped us to improve the presentation.

Author Contributions All authors contributed to the study conception and design. Material preparation, data collection and analysis were performed by all authors. The first draft of the manuscript was written by Nikolaos S. Papageorgiou and all authors commented on previous versions of the manuscript. The authors read and approved the final manuscript.

Funding The authors have been supported by the Slovenian Research Agency grants P1-0292, J1-8131, N1-0064, N1-0083, and N1-0114. The work of Vicenţiu D. Rădulescu was supported by a grant of the Romanian Ministry of Education and Research, CNCS-UEFISCDI, project number PN-III-P4-ID-PCE-2020-0068, within PNCDI III.

Availability of data and material All data generated or analysed during this study are included in this article.

\section{Declarations}

Consent to participate All authors consent to participate to this work.

Consent for publication All authors consent for publication.

Conflict of interest The authors declare that they have no conflict of interest.

Open Access This article is licensed under a Creative Commons Attribution 4.0 International License, which permits use, sharing, adaptation, distribution and reproduction in any medium or format, as long as you give appropriate credit to the original author(s) and the source, provide a link to the Creative Commons licence, and indicate if changes were made. The images or other third party material in this article are included in the article's Creative Commons licence, unless indicated otherwise in a credit line to the material. If material is not included in the article's Creative Commons licence and your intended use is not permitted by statutory regulation or exceeds the permitted use, you will need to obtain permission directly from the copyright holder. To view a copy of this licence, visit http://creativecommonshorg/licenses/by/4.0/.

\section{References}

1. Bahrouni, A., Rădulescu, V.D., Repovš, D.D.: A weighted anisotropic variant of the Caffarelli-KohnNirenberg inequality and applications. Nonlinearity 31, 1516-1534 (2018)

2. Bahrouni, A., Rădulescu, V.D., Repovš, D.D.: Double phase transonic flow problems with variable growth: nonlinear patterns and stationary waves. Nonlinearity 32, 2481-2495 (2019) 
3. Bahrouni, A., Rădulescu, V.D., Winkert, P.: Double phase problems with variable growth and convection for the Baouendi-Grushin operator. Z. Angew. Math. Phys. 71(6), 15 (2020). Paper No. 183

4. Ball, J.M.: Convexity conditions and existence theorems in nonlinear elasticity. Arch. Rational Mech. Anal. 63(4), 337-403 (1976/77)

5. Ball, J.M.: Discontinuous equilibrium solutions and cavitation in nonlinear elasticity. Philos. Trans. Roy. Soc London Ser. A 306(1496), 557-611 (1982)

6. Baroni, P., Colombo, M., Mingione, G.: Harnack inequalities for double phase functionals. Nonlinear Anal. 121, 206-222 (2015)

7. Baroni, P., Colombo, M., Mingione, G.: Regularity for general functionals with double phase. Calc. Var. Partial Differ. Equ. 57 Art 62 (2018)

8. Benci, V., D’Avenia, P., Fortunato, D., Pisani, L.: Solitons in several space dimensions: Derrick's problem and infinitely many solutions. Arch. Ration. Mech. Anal. 154, 297-324 (2000)

9. Byun, S.S., Ko, E.: Global $C^{1, \alpha}$ regularity and existence of multiple solutions for singular $p(x)$ Laplacian equations. Calc. Var. Partial Differ. Equ. 56, 76 (2017)

10. Cahn, J.W., Hilliard, J.E.: Free energy of nonuniform system I: interfacial free energy. J. Chem. Phys. 28, 258-267 (1958)

11. Cherfils, L., Ilyasov, Y.: On the stationary solutions of generalized reaction-diffusion equations with $p$ \& $q$-Laplacian. Comm. Pure Appl. Anal. 4, 9-22 (2005)

12. Colombo, M., Mingione, G.: Regularity for double phase variational problems. Arch. Ration. Mech. Anal. 215, 443-496 (2015)

13. De Filippis, C., Mingione, G.: On the regularoty of minima of non-autonoumous functionals. J. Geom. Anal. 30, 1584-1626 (2020)

14. Diening, L., Harjulehto, P., Hästo, P., Ruzička, M.: Lebesgue and Sobolev Spaces with Variable Exponents, Lecture Notes in Mathematics, vol. 2017. Springer, Heidelberg (2011)

15. Fan, X.: Global $C^{1, \alpha}$ regularity for variable exponent elliptic equations in divergence form. J. Differ. Equ. 235, 397-417 (2007)

16. Gasinski, L., Papageorgiou, N.S.: Anisotropic nonlinear Neumann problems. Calc. Var. Partial Differ. Equ. 42, 323-354 (2011)

17. Ghergu, M., Rădulescu, V.D.: Singular Elliptic Problems: Bifurcation and Asymptotic Analysis, Oxford Lecture Series in Mathematics and Its Applications, vol. 37. Oxford University Press, Oxford (2008)

18. Giacomoni, J., Schindler, I., Takač, P.: Sobolev versus Hölder minimizers and existence of multiple solutions for a singular quasilinear equation. Ann. Scu. Norm. Super Pisa 6, 117-158 (2007)

19. Hu, S., Papageorgiou, N.S.: Handbook of Multivalued Analysis. Volume I: Theory. Kluwer Academic Publishers, Dodrecht (1997)

20. Li, G., Yang, C.: The existence of a nontrivial solution to a nonlinear elliptic boundary value problem of $p$-Laplacian type without Ambrosetti-Rabinowitz condition. Nonlinear Anal. 72, 4602-4613 (2010)

21. Lieberman, G.: The natural generalization of the natural conditions of Ladyzhenskaya and Uraltseva for elliptic equations, Comm. Partial Differ. Equ. 16, 311-361 (1991)

22. Marcellini, P.: On the definition and the lower semicontinuity of certain quasiconvex integrals. Ann. Inst. H. Poincaré, Anal. Non Linéaire 3, 391-409 (1986)

23. Marcellini, P.: Regularity and existence of solutions of elliptic equations with $p, q$-growth conditions. J. Differ. Equ. 90, 1-30 (1991)

24. Marcellini, P.: Everywhere regularity for a class of elliptic systems without growth conditions. Ann. Scuola Norm. Sup. Pisa Cl. Sci. (4) 23(1), 1-25 (1996)

25. Marcellini, P.: Growth conditions and regularity for weak solutions to nonlinear elliptic PDEs, J. Math. Anal. Appl. https://doi.org/10.1016/j.jmaa.2020.124408

26. Morrey, C.h.B. Jr.: Multiple Integrals in the Calculus of Variations. Reprint of the 1966 edition, Classics in Mathematics. Springer, Berlin (2008)

27. Papageorgiou, N.S., Scapellato, A.: Constant sign and nodal solutions for parametric $(p, 2)$-equations. Adv Nonlinear Anal. 9(1), 449-478 (2020)

28. Papageorgiou, N.S., Winkert, P.: Singular $p$-Laplacian equations with superlinear perturbation. J. Differ. Equ. 266, 1462-1487 (2019)

29. Papageorgiou, N.S., Winkert, P.: Applied Nonlinear Functional Analysis. De Gruyter, Berlin (2018)

30. Papageorgiou, N.S., Zhang, C.: Noncoercive resonant $(p, 2)$-equations with concave terms. Adv. Nonlinear Anal. 9(1), 228-249 (2020)

31. Papageorgiou, N.S., Vetro, C., Vetro, F.: Positive solutions for $\operatorname{singular}(p, 2)$-equations. Z. Angew. Math. Phys. 70:72, 1-10 (2019)

32. Papageorgiou, N.S., Rădulescu, V.D., Repovš, D.: Nonlinear Analysis-Theory and Methods. Springer Monographs in Mathematics. Springer, Cham (2019) 
33. Papageorgiou, N.S., Rădulescu, V.D., Repovš, D.D.: Double-phase problems and a discontinuity property of the spectrum. Proc. Am. Math Soc. 7, 2899-2910 (2019)

34. Papageorgiou, N.S., Rădulescu, V.D., Repovš, D.D.: Positive solutions for nonlinear parametric singular Dirichlet problems. Bull. Math. Sci. 9(3), 1950011 (2019). 21 pp

35. Papageorgiou, N.S., Rădulescu, V.D., Repov̌̌, D.D.: Ground state and nodal solutions for a class of double phase problems. Z. Angew. Math. Phys. 71(1)Paper No. 15 (2020)

36. Papageorgiou, N.S., Rădulescu, V.D., Repovš, D.D.: Nonlinear nonhomogeneous singular problems. Calc Var. Partial Differ. Equ. 59, 9 (2020)

37. Papageorgiou, N.S., Rădulescu, V.D., Repov̌̌, D.: Anisotropic equations with indefinite potential and competing nonlinearities. Nonlinear Anal. 201(111861), 24 (2020)

38. Ragusa, M.A., Tachikawa, A.: Regularity for minimizers for functionals of double phase with variable exponents. Adv. Nonlinear Anal. 9(1), 710-728 (2020)

39. Rădulescu, V.D., Repov̌̌, D.D.: Partial Differential Equations with Variable Exponents: Variational Methods and Qualitative Analysis. CRC Press, Boca Raton (2015)

40. Saoudi, K., Ghanmi, A.: A multiplicity result for a singular equation involving the $p(x)$-Laplace operator. Complex Var. Elliptic Equ. 62, 695-725 (2017)

41. Zhang, Q.: A strong maximum principle for differential equations with nonstandard $p(x)$-growth conditions. J. Math. Anal. Appl. 312, 125-143 (2005)

42. Zhang, Q., Rădulescu, V.D.: Double phase anisotropic variational problems and combined effects of reaction and absorption terms. J. Math. Pures Appl. 118, 159-203 (2018)

43. Zhikov, V.V.: Averaging of functionals of the calculus of variations and elasticity. Math. USSR-Izves. 29, 33-66 (1987)

44. Zhikov, V.V.: On variational problems and nonlinear elliptic equations with nonstandard growth conditions. J. Math. Sci. 173, 463-570 (2011)

Publisher's Note Springer Nature remains neutral with regard to jurisdictional claims in published maps and institutional affiliations. 